\title{
Uncovering a neural circuit controlling adult quiescent neural stem cell activation in the subventricular zone
}

\section{Authors/Affiliations:}

Moawiah M Naffaa ${ }^{1,2, \dagger}$, Rehan R Khan ${ }^{2}$, Chay T. Kuo, Henry H. Yin ${ }^{1,3, \dagger}$

1. Department of Psychology and Neuroscience; Duke University

2. Department of Cell Biology, Duke University, School of Medicine

3. Department of Neurobiology; Duke University School of Medicine

\section{†Correspondence:}

Moawiah Naffaa moawiah.naffaa@duke.edu

Henry Yin hy43@duke.edu

\section{Summary}

The maintenance and differentiation of the adult neural stem cells (NSCs) in the subventricular zone (SVZ) are controlled by cell-intrinsic molecular pathways that interact with extrinsic signaling cues. How neurogenesis in the SVZ is regulated by neural circuit activity remains poorly understood. Here we identified a novel neural circuit that regulates the state of lateral ventricular wall (LV) NSCs. Our results demonstrate that direct glutamatergic inputs from the frontal cortex, as well as local inhibitory interneurons, control the activity of subependymal cholinergic neurons. In vivo optogenetic and chemogenetic stimulation of defined neuronal populations within this circuit were sufficient to control LV NSC proliferation and SVZ neurogenesis. Moreover, acetylcholine (Ach), which activates $\mathrm{M}_{1}$ muscarinic ACh receptors, triggers the activation of quiescent NSCs. These findings shed light on neural activity-dependent regulation of postnatal and adult LV NSCs activation and SVZ neurogenesis. 
The rodent subventricular zone (SVZ) of the lateral ventricles (LV) is a major site of postnatal neurogenesis ${ }^{1}$. SVZ neurogenesis provides a useful model system to study neuronal regeneration and tissue remodeling in the adult brain ${ }^{2,3}$. The LV neural stem cells (LV NSCs) exist in either a quiescent (qNSCs) or an actively dividing (aNSCs) state ${ }^{4,5}$. Most of LV NSCs are quiescent adult cells, but upon activation they divide asymmetrically for self-renewal or differentiate to become transient amplifying intermediate progenitors (TAPs) ${ }^{4,6,7}$. Studies have shown that TAPs can divide and differentiate into doublecortin-positive neuroblasts ${ }^{8,9}$, which migrate to the olfactory bulb (OB) $8,10,11$, where they become mature interneurons that are incorporated into the local circuitry ${ }^{12,13}$. The generation of adult-born neurons from the SVZ niche contributes to experience-dependent plasticity in the postnatal brain ${ }^{14,15}$ and plays a critical role in social behavior in rodents $13,16,17$.

In postnatal and adult rodents, most LV NSCs are qNSCs ${ }^{4,5,7}$. The specific input signals that promote activation of qNSCs remain largely unknown. LV NSCs receive inputs from a variety of sources, including neighboring NSCs, TAPs, and neuroblasts ${ }^{18,19}$. Different neurotransmitters, including GABA, dopamine, and serotonin, released by neurons in different brain regions, are also known to regulate postnatal and adult SVZ neurogenesis ${ }^{20-25}$. However, little is known about the mechanisms by which synaptic inputs influence SVZ neurogenesis.

Recent work has identified a small population of cholinergic neurons exists in the subependymal space to the lateral ventricle. These neurons, which express Choline acetyltransferase $\left(\mathrm{ChAT}^{+}\right)$, can modulate the proliferation of LV NSCs and neuroblasts in an activity-dependent manner ${ }^{26}$. They are distinct from neighboring striatal cholinergic interneurons, and release acetylcholine (Ach) into the SVZ niche. We hypothesize that this small population of subependymal $\mathrm{ChAT}^{+}$(subep-ChAT ${ }^{+}$) neurons is a key node in the neural circuit regulation of $\mathrm{LV}$ NSCs. Using a circuit-tracing strategy, we identified a novel neural circuit involving anterior cingulate glutamatergic projections and local calretinin-positive interneurons that can regulate the activity of subep-ChAT ${ }^{+}$neurons. In turn, ACh from subep-ChAT ${ }^{+}$neeurons modulates the activity of LV qNSCs by activating $\mathrm{M}_{1}$ muscarinic receptors. These findings revealed a novel circuit mechanism by which frontal cortical inputs can influence cholinergic signaling in the SVZ niche to regulate the activity of LV qNSCs. 


\section{Results}

\section{Excitatory and inhibitory inputs to the subep-ChAT ${ }^{+}$neurons}

Unlike striatal $\mathrm{ChAT}^{+}$neurons, Subep-ChAT ${ }^{+}$neurons do not show tonic activity in the absence of synaptic inputs, suggesting excitatory synaptic inputs are needed to activate these neurons ${ }^{27}$. However, it is currently unknown where the excitatory inputs come from and how they influence the activity of Subep-ChAT ${ }^{+}$neurons. We hypothesized that glutamatergic inputs from cortical projection neurons may provide excitatory drive to subep-ChAT ${ }^{+}$neurons. Since cortical neurons express vesicular glutamate transporter 1 (Vglut1), to selectively stimulate these glutamatergic inputs, we crossed Vglut1-Cre mice with ChAT-eGFP, and ChR2-tdTomato transgenic mouse line $(A i 27)$ that expresses the excitatory opsin channelrhodopsin in a Credependent manner ${ }^{28}$. The resulting Vglut1-Cre::ChAT-eGFP::Ai27 express channelrhodopsin in VGlut $1^{+}$neurons (Fig. 1A).

Whole-cell recordings from subep-ChAT ${ }^{+}$neurons from $\mathrm{P} 35$ mice showed robust firing in

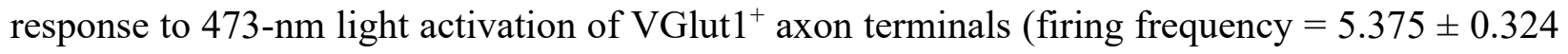
$\mathrm{Hz})$ (Fig. 1B). In voltage-clamp mode $(-60 \mathrm{mV})$, photo-stimulation evoked excitatory postsynaptic currents (EPSCs) in subep-ChAT ${ }^{+}$neurons. These currents can be blocked by glutamatergic receptors antagonists AP5 (NMDA) and CNQX(AMPA) (Current amplitude $=98.2 \pm 10.7$ pA, and current latency $=8.6 \pm 0.62 \mathrm{~ms}$ ) (Fig. 1C). Thus, we demonstrate the existence of cortical glutamatergic inputs that drive subep-ChAT ${ }^{+}$neurons.

To determine the inhibitory inputs to subep-ChAT ${ }^{+}$neurons, we expressed ChR2 in GABAergic neurons by crossing vesicular GABA transporter (Vgat-Cre) mice, ChAT-eGFP, and Ai27 mice. The resulting Vgat-Cre::ChAT-eGFP::ChR2 mice allows us to record from subep$\mathrm{ChAT}^{+}$neurons in animals with ChR expression in GABAergic neurons (Extended Fig. 1A). Activating $\mathrm{VGAT}^{+}$neurons via 473-nm laser for $0.5 \mathrm{~s}$ and holding at $-60 \mathrm{mV}$ resulted in consistent IPSCs in subep-ChAT ${ }^{+}$neurons, which was blocked by picrotoxin (GABA $\mathrm{G}_{\mathrm{A}}$ receptors antagonist) (Current amplitude $=196.1 \pm 24.13 \mathrm{pA}$, and current latency $=4.5 \pm 0.88 \mathrm{~ms}$ ) (Extended Fig.1B). This suggests that subep-ChAT ${ }^{+}$neurons receive direct GABAergic inputs, which can suppress the release of ACh into LV NSCs. 


\section{Distinct connectivity of subep-ChAT ${ }^{+}$neurons and striatal $\mathrm{ChAT}^{+}$neurons}

Our initial electrophysiological results established the presence of excitatory and inhibitory inputs to the subep-ChAT ${ }^{+}$neurons, and their physiological effects. However, it remains unclear exactly where these inputs originate. To answer this question, we generated and validated a Credependent R26R-FLEX-TVA-2A-RabiesG-2A-tdTomato-FLEX (R26F-RTT) mice to trace the connectivity (Fig. 1D and Extended Fig. 2A). To allow for monosynaptic tracing of (ChAT-Cre)targeted neurons via a single EnvA G-deleted Rabies-eGFP viral injection, we crossed ChATCre::R26F-RTT mice to produce mice that express the TVA receptor, Rabies G protein, and tdTomato in cholinergic neurons (Fig. 1E). Injection of EnvA G-deleted rabies-eGFP virus into the striatum of P30 ChAT-Cre::R26F-RTT mice efficiently labeled striatal $\mathrm{ChAT}^{+}$neurons (Extended Fig. 2B and C). As the striatal $\mathrm{ChAT}^{+}$neurons receive synaptic inputs from each other ${ }^{29}$, an infection of a striatal $\mathrm{ChAT}^{+}$neuron with EnvA G-deleted Rabies-eGFP virus results in labeling other striatal $\mathrm{ChAT}^{+}$neurons, which subsequently pass rabies to other connected neurons (Extended Fig. 2D).

We injected EnvA G-deleted Rabies-eGFP virus into the lateral ventricles to target subep$\mathrm{ChAT}^{+}$neurons, immediately following mannitol injection to disrupt the ependymal layer locally (Fig. 1F). Using this strategy, we were able to trace the source of monosynaptic inputs to subep$\mathrm{ChAT}^{+}$neuron (Fig. 1G). Unlike striatal $\mathrm{ChAT}^{+}$neurons, subep-ChAT ${ }^{+}$neurons in the $\mathrm{SVZ}$ niche are not connected with neighboring ChAT + neurons, or with striatal $\mathrm{ChAT}^{+}$neurons (Fig. H). This result confirms that subep-ChAT ${ }^{+}$neurons have distinct neuronal connectivity.

\section{Local inhibitory input to subep-ChAT ${ }^{+}$neurons}

As we injected EnvA G-deleted Rabies-eGFP virus into the lateral ventricles of P30 ChATCre::R26F-RTT mice to infect subep-ChAT ${ }^{+}$neurons, local $\mathrm{GFP}^{+}$interneurons were observed (Fig. 2A and B). While staining for markers of the known inhibitory neurons, we noticed colocalization between $\mathrm{GFP}^{+}$interneurons and calretinin $\left(\mathrm{CR}^{+}\right)$antibody (Fig. 2B).

To test if the $\mathrm{CR}^{+}$interneurons provide local inhibitory inputs to subep-ChAT $\mathrm{T}^{+}$neurons, we performed whole-cell patch clamp recording from subep-ChAT ${ }^{+}$neurons of P30 $\mathrm{Cr}$ Cre::ChAT-eGFP::ChR2 mice (Fig. 2C). A high-chloride internal solution was used to detect 
inhibitory postsynaptic current (IPSC) while holding at $-60 \mathrm{mV}$ in voltage clamp. We stimulated local $\mathrm{CR}^{+}$interneurons with blue light and revealed robust evoked IPSCs, which were completely blocked by picrotoxin (current amplitude $=122.3 \pm 9.0 \mathrm{pA}$, and current latency $=4.9 \pm 0.33 \mathrm{~ms}$ ) (Fig. 2D). We then tested for other known inhibitory interneurons expressing calbindin $\left(\mathrm{CB}^{+}\right)$, somatostatin $\left(\mathrm{SST}^{+}\right)$or parvalbumin $\left(\mathrm{PV}^{+}\right)$(Extended Fig. 3A). Using whole-cell recording from subep-ChAT ${ }^{+}$neurons, an optogenetic stimulation of GABA released from these other inhibitoryinterneurons did not produce IPSCs (Extended Fig. 3B).

To further confirm the local $\mathrm{CR}^{+}$interneuron inputs to the subep-ChAT ${ }^{+}$neurons, we used a Patterned Light Stimulator LED controller (Mightex) to target $\mathrm{CR}^{+}$labelled neurons in SVZ wholemounts from P35 Cr-Cre::ChAT-eGFP::ChR2 mice. Focal optogenetic activation of a single $\mathrm{CR}^{+}$interneuron generated IPSCs in subep-ChAT ${ }^{+}$neuron (current amplitude $=40.14 \pm$ $3.78 \mathrm{pA}$, and current latency $=4.7 \pm 0.4 \mathrm{~ms}$ ) (Fig. $2 \mathbf{E}$ and F). Together these results confirm that local $\mathrm{CR}^{+}$interneurons are the main source of inhibitory inputs to the subep-ChAT ${ }^{+}$neuron. Subsequently, we stained against $\mathrm{CR}$ and ChAT using P30 C57BL/6J mice, revealing the presence of 2-3 $\mathrm{CR}^{+}$interneurons surrounding each subep-ChAT ${ }^{+}$neuron (Fig. 2G and Fig.4 G).

\section{Excitatory inputs from cingulate cortex area 1 (Cg1) to subep-ChAT ${ }^{+}$neurons}

The commonly used EnvA G-deleted Rabies-eGFP virus does not illuminate long distance connections for cholinergic neurons in the brain. We then tried a variety of modified rabies viruses from different laboratories and found that the rVSV (RABV-G)-eGFP virus labeled distal GFP ${ }^{+}$ projection neurons from the anterior cingulate cortex ( $\mathrm{Cg} 1$ region) (Fig. 3A and $\mathbf{B})^{30}$. To confirm $\mathrm{Cg} 1$ input to subep-ChAT ${ }^{+}$neurons, we first injected AAV-CaMKII-hChR2(E123A)-mCherry virus into the $\mathrm{Cg} 1$ region of $\mathrm{P} 30 \mathrm{C} 57 \mathrm{BL} / 6 \mathrm{~J}$ mice to express mCherry in the $\mathrm{GFP}^{+}$neurons from Fig. 3B (Fig. 3C). By tracing projections in SVZ region, we observed them adjacent to the subep$\mathrm{ChAT}^{+}$neurons (Fig. 3D).

To functionally validate a monosynaptic input from $\mathrm{Cg} 1$ to the subep-ChAT ${ }^{+}$neurons, we injected a Cre-dependent ChR2 viral vector (pAAV-Ef1a-DIO hChR2(E123T/T159C)-EYFP) into the $\mathrm{Cg} 1$ region of $\mathrm{P} 30$ Chat-Cre::Ai9 mice to express ChR2 (Fig. 3E). Optogenetic stimulation of cingulate projections reliably evoked action potentials in subep-ChAT ${ }^{+}$neurons (firing frequency $=2.5 \pm 0.327 \mathrm{~Hz}$ ) (Fig. 3F). As shown in Fig. 3G, using voltage clamp, we reliable evoked EPSCs, 
which were blocked completely by the glutamate receptor antagonists AP5 (NMDA) and CNQX $($ AMPA) (current amplitude $=64.86 \pm 2.424 \mathrm{pA}$, and current latency $=9.1 \pm 0.7 \mathrm{~ms})$. This confirms the presence of glutamatergic neurons in the ipsilateral $\mathrm{Cg} 1$ region that excite subep-ChAT ${ }^{+}$ neurons.

In addition, we injected pAAV-Ef1a-DIO hChR2(E123T/T159C)-mCherry virus into the Cg1 region of $\mathrm{P} 30$ Vglut1-Cre::ChAT-eGFP mice to express ChR2 in Vglut ${ }^{+}$neurons (Extended Fig. 4A). Again we found reliably evoked EPSCs upon optogenetic stimulation. These currents were blocked completely by AP5 and CNQX, confirming the presence of glutamatergic inputs (current amplitude $=53.29 \pm 2.96 \mathrm{pA}$, and current latency $=8.7 \pm 0.44 \mathrm{~ms}$ ) (Extended Fig. 4B). These results show that a specific population of cortical neurons in $\mathrm{Cg} 1$ region provides excitatory drive to subep-ChAT ${ }^{+}$neurons (Fig. 4G).

\section{Calretinin-Cre (Cr-Cre) mice label the distal excitatory neuronal inputs from $\mathrm{Cg} 1$ to the subep-ChAT ${ }^{+}$neurons}

After injecting pAAVrg-hSyn-DIO hM3D(Gq)-mCherry virus to the SVZ of P30 Cr-Cre mice, we observed $\mathrm{mCherry}^{+} \mathrm{CR}^{+}$neurons labeled in $\mathrm{Cg} 1$ region. These $\mathrm{CR}^{+}$neurons are similar in morphology and location to neurons that directly excite subep-ChAT ${ }^{+}$neurons (Fig. 4A and B). To determine if they are the same, we injected pAAV-Ef1a-DIO-hChR2(E123T/T159C)-mCherry into the $\mathrm{Cg} 1$ region of $\mathrm{P} 30 \mathrm{Cr}$-Cre mice, we observed processes of $\mathrm{Cg} 1 \mathrm{CR}^{+}$neurons in the lateral wall of the lateral ventricle adjacent to the subep-ChAT ${ }^{+}$neurons (Fig. 5A, B \& G).

We performed whole-cell recording from subep-ChAT ${ }^{+}$neurons in P35 Cr-Cre::ChATeGFP::ChR2 mice (Fig. 4C). Recording in voltage mode at $-60 \mathrm{mV}$, optogenetic stimulation of terminals $\mathrm{CR}^{+}$neurons reliably evoked EPSCs (Current amplitude $=106 \pm 6.68 \mathrm{pA}$, and current latency $=5.13 \pm 0.44 \mathrm{~ms}$ ) (Fig. 4D). Together these results show that a small population of $\mathrm{CR}^{+}$ neurons in $\mathrm{Cg} 1$ is the source of glutamatergic inputs to subep-ChAT+ neurons. To functionally test if the excitatory $\mathrm{Cg} 1 \mathrm{CR}^{+}$neurons regulate subep-ChAT ${ }^{+}$neurons, we injected AAV-EF1aDIO-hChR2(E123T/T159C)-mCherry virus into the $\mathrm{Cg} 1$ region of P30 Cr-Cre::ChAT-eGFP mice (Fig. 4F). Whole-cell recording from subep-ChAT ${ }^{+}$neurons in voltage clamp mode showed evoked EPSCs (Current amplitude $=33 \pm 2.46 \mathrm{pA}$, and latency $=5.13 \pm 0.44 \mathrm{~ms}$ ) (Fig. 4F). 
The total number of labeled $\mathrm{Cg} 1$ cortical neurons with $\mathrm{Cr}$-Cre mice is must lower than the number of labeled neurons with Vglut-Cre mice. These results together confirmed that $\mathrm{Cr}$-Cre mouse is a reliable tool to study a subpopulation of glutamatergic neurons from the $\mathrm{Cg} 1$ region of the activity of subep-ChAT ${ }^{+}$neurons.

\section{Manipulation of subep-ChAT ${ }^{+}$in vivo controls LV NSCs proliferation and SVZ neurogenesis}

Having determined the projection from $\mathrm{Cg} 1$ region to subep-ChAT ${ }^{+}$neurons, we next studied the functional role of this projection in vivo. In particular, we tested the hypothesis that this excitatory input to subep-ChAT ${ }^{+}$neurons could regulate the LV NSCs proliferation and SVZ neurogenesis. To accomplish this aim, we injected AAV-EF1a-DIO-hChR2 (E123T/T159C)mCherry virus and implanted an optical fiber into the $\mathrm{Cg} 1$ region of $\mathrm{P} 30 \mathrm{Cr}$-Cre mice. Stimulation of $\mathrm{Cg} 1 \mathrm{CR}^{+}$neurons were conducted continuously for three days and were delivered by TTL control of 473-nm laser, $10 \mathrm{~ms}$ pulses at $10 \mathrm{~Hz}$, lasting $10 \mathrm{~s}$, given once every $1 \mathrm{~min}$ (Fig. $\mathbf{5 A}$ and B).

After 3 days, the brains were removed and brain slices of the stimulated $\mathrm{Cg} 1$ sections were stained against ChAT and DCX antibodies. We quantified activity of subep-ChAT ${ }^{+}$neurons and on the ipsilateral (activation) side comparing to contralateral (control) side. There was significant increase in the expression level of ChAT protein as indicated by GFP in the ipsilateral SVZ (activation) compared to the contralateral SVZ (control) (109.4 $\pm 19.62 \%)$ (Fig. 5C \& D). Higher ChAT expression indicates more ACh synthesis and release by the subep-ChAT ${ }^{+}$neurons when activated by the $\mathrm{Cg} 1 \mathrm{CR}^{+}$neurons, and further confirms our previous tracing and functional studies. We also observed an increase in the number of $\mathrm{DCX}^{+}$neuroblasts in the ipsilateral SVZ (activation) in contrast to the contralateral SVZ (control) $(23.42 \pm 5.42 \%)$ (Fig. 5E \& F). These results strongly suggest that the excitatory $\mathrm{Cg} 1 \mathrm{CR}^{+}$neurons modulate neurogenesis in $\mathrm{SVZ}$ niche by regulating the neuronal activity of subep-ChAT ${ }^{+}$and releasing ACh into LV NSCs.

We performed a similar in vivo optogenetic stimulation experiment where $\mathrm{Cg} 1 \mathrm{CR}^{+}$ neurons were stimulated for $36 \mathrm{hrs}$ only. This time, the stimulated sections were stained against Ki67, a proliferation marker, to quantify the number of proliferative cells after circuit stimulation (Fig. 5H). The results showed an increase in the number of proliferative cells $\left(\mathrm{Ki}^{+} 7^{+}\right)$in the SVZ 
niche of ipsilateral side compared to the contralateral side $(26.77 \pm 6.43 \%)$ (Fig. 5I \&J). This further supports that glutamatergic $\mathrm{CR}^{+}$neurons in the cingulate cortex modulate neurogenesis by regulating the release of $\mathrm{ACh}$ from subep-ChAT ${ }^{+}$neurons into the LV NSCs.

We further tested the effect of $\mathrm{CR}^{+}$neurons stimulation on SVZ neurogenesis using chemogenetics (DREADDs). For this experiment, pAAV-hSyn-DIO-hM3D(Gq)-mCherry virus was injected into $\mathrm{Cg} 1$ region of P30 Cr-Cre mice as described in the experimental design (Extended Fig. 5A). To activate $\mathrm{Cg} 1 \mathrm{CR}^{+}$neurons, clozapine (CNO) was injected intraperitoneally for five days. The CNO-mediated chemogenetics activation of $\mathrm{Cg} 1 \mathrm{CR}^{+}$neurons revealed an increase in the number of $\mathrm{DCX}^{+}$neuroblasts in the ipsilateral $\mathrm{SVZ}$ compared to the contralateral SVZ $(30.8 \pm 5.95 \%)$ (Extended Fig. 5B \& C). These results are comparable with that were seen in the in vivo optogenetic stimulation experiment of subep-ChAT ${ }^{+}$circuit, confirming increased neurogenesis after circuit stimulation.

\section{ACh modulates LV NSCs proliferation and SVZ neurogenesis via activating qNSCs}

As the stimulation of subep-ChAT ${ }^{+}$circuit for $36 \mathrm{hrs}$ increased the number of in vivo proliferative cells, we hypothesized that ACh triggers the activation of LV qNSCs, which are usually in the quiescent state in vivo ${ }^{31}$. To test this hypothesis, we performed an in vivo optogenetic stimulation experiment to stimulate (Cg1-subep-ChAT $\left.{ }^{+}\right)$circuit for 12 hrs (Fig. 5K). Epidermal growth factor (EGF) is a key growth factor for proliferation of LV NSCs ${ }^{32}$. Upregulation of EGF receptors (EGFRs) is an important early indicator of LV qNSC activation ${ }^{39}$. Therefore we measured the number of NSCs with phosphorylated EGF receptors (pEGFRs) as a result of stimulation. As the stimulated sections were stained against pEGFRs(PhosphoY 1068) protein, an increase in number of $\mathrm{pEGFR}^{+} \mathrm{NSCs}$ were noticed in ipsilateral SVZ compared to the contralateral SVZ $(48.5 \pm 8.2 \%$ ) (Fig. 5L, M \& N). This fast upregulation of pEGFRs suggests that (Cg1 subep-ChAT ${ }^{+}$) circuit promotes LV NSCs proliferation by activating LV qNSCs.

To determine how ACh initiates the NSCs proliferation in SVS niche, we studied the effect of adding carbachol (an analogue of ACh) to the early differentiating SVZ NSCs culture on the quantity of produced neuroblast $\left(\mathrm{DCX}^{+}\right)$cells. We noticed an increase in the number of $\mathrm{DCX}^{+}$ cells in carbachol-treated samples compared to control samples in the second- and third-day 
sample collections (Day 2: $24.2 \pm 6.63 \%$ ) (Day 3: $43.4 \pm 9.1 \%$ ) (Fig. 6A and B). The initial increase in the number of neuroblasts may indicate that adding carbachol triggers an earlier differentiation of SVZ NSCs cells. To further look at the effect of adding carbachol to SVZ NSCs culture, we stained control and carbachol-treated samples for two days in the differentiation media using DCX and GFAP antibodies. We found a significant increase in the number of $\mathrm{DCX}^{+}$cells in carbachol-treated samples compared to the control samples (63.40 $\pm 15.25 \%)$ (Extended Fig. 6A and B). However, the number of $\mathrm{GFAP}^{+}$cells were not significantly changed in carbachol-treated samples compared to the control samples (Extended Fig. 6A and C). GFAP protein is known to be expressed by SVZ qNSCs and aNSCs ${ }^{33}$. This could explain the comparable total number of qNSCs and aNSCs in control and carbachol-treated samples. As carbachol-treated samples have no significant effect on the number of $\mathrm{GFAP}^{+}$cells, the data suggests that $\mathrm{ACh}$ activates SVZ qNSCs.

In addition, we studied the effect of adding carbachol to SVZ NSCs by quantifying pEGFRs in the proliferation media. During the proliferation stage, some of the NSCs are quiescent. Activated-NSCs upregulate EGFRs, which become activated via phosphorylation as EGF binds to them ${ }^{34,35}$. We added carbachol in a fresh proliferation media to SVZ NSCs culture and collected the samples after 12 hrs. Carbachol-treated samples expressed more pEGFRs(PhosphoY1068) protein than control samples $(72 \pm 14.8 \%)$. This suggests that ACh activates qNSCs which results in a phosphorylation of more EGFRs(pEGFRs) (Fig. 6C and D). To test if the upregulation of pEGFRs protein in carbachol-treated samples is related to qNSCs activation, we performed similar experiment to look at the number of $\mathrm{Ki}_{6} 7^{+}$cells and $\mathrm{EdU}^{+} / \mathrm{Ki} 67^{+}$cells ratio in carbachol-treated samples compared to control samples (Fig. 6E). Although the difference in the number of $\mathrm{Ki}^{+} 7^{+}$ cells between carbachol-treated samples and control samples was insignificant, the $\mathrm{EdU}^{+} / \mathrm{Ki}^{+}$ cells ratio was notably higher in carbachol-treated samples $(22.8 \pm 4.5 \%)$ (Fig. 6E, F and G). Altogether these results suggest that NSCs are driven out of quiescence to become activated in carbachol-treated samples. It is also possible that the new $\mathrm{EdU}^{+}$cells in carbachol-treated samples are the newly activated NSCs, which are not upregulating Ki67 protein yet.

To understand how ACh promotes the proliferation of NSCs, we studied the effect of carbachol on pEGFR protein in the early stage of SVZ NSCs differentiation. By treating cells with carbachol once at the beginning of the differentiation stage, we noticed an increase in the quantity 
of pEGFR (PhosphoY1068) protein in carbachol-treated samples collected 12 hrs post incubation $(31.53 \pm 8.4 \%, \mathrm{n}=4, \mathrm{P}=0.0095, \mathrm{t}(6)=3.75$, Unpaired $\mathrm{t}$ test) (Fig. 6H and I). However, the samples that were collected after 12 hrs did not show a significant difference between carbacholtreated and control ones (Fig. 6I). The addition of carbachol at this early differentiation stage resulted in more proliferative cells, suggesting more qNSCs became activated in treated samples compared to control ones.

We also added carbachol for one day and measured the number of proliferative cells in SVZ NSCs culture during the early differentiation stage. There was a significant increase in the number of $\mathrm{Ki}^{+}$cells $(11.9 \pm 2.9 \%)$ (Fig. 6J and K), and $\mathrm{EdU}^{+} / \mathrm{Ki}^{+} 7^{+}$cells ratio $(18.94 \pm 4.74 \%)$

(Fig. 6J and $\mathbf{L}$ ) in carbachol-treated samples in comparison to the control samples. This suggests that activated qNSCs in carbachol-treated samples upregulate $\mathrm{Ki}^{+} 7^{+}$protein to proliferate and differentiate into highly proliferative TACs. In a similar experimental condition, we noticed a significant increase in the quantity of pEGFR (PhosphoY1068) protein in carbachol-treated samples compared to control samples $(86.91 \pm 20.32 \%)$ (Extended Fig. 6D and E).

\section{ACh modulates LV qNSCs via activating muscarinic $1\left(M_{1}\right)$ receptors}

So far, our results have suggested that ACh activates LV qNSCs to initiate proliferation and promote neurogenesis. To further test this hypothesis, we explored the cholinergic receptors which are expressed in the LV qNSCs. Therefore, we performed an RNA-seq experiment for SVZ NSCs cultures in the proliferation media incubated with carbachol for one day compared to control samples (Extended Fig. 7A and B). Although the ChRM1 gene expression was detected in a relatively low expression level in control samples, it was significantly decreased in the carbacholtreated samples compared to control ones. This also was confirmed by the quantitative reverse transcription PCR (RT-qPCR) analysis (Extended Fig. 7C and D). These results suggest that ChRM1 genes and $\mathrm{M}_{1}$ receptors are present in the LV qNSCs state. To check this, we performed immunofluorescence (IF) staining of wholemount SVZ using anti $\mathrm{M}_{1}$ receptors (by using ChRM1 antibody) and VCAM 1(Vascular cell adhesion protein 1; a marker for qNSCs in SVZ) antibodies. On imaging, we observed that the VCAM $1^{+}$SVZ NSCs express $M_{1}$ receptors (Fig. 7A), confirming that $\mathrm{M}_{1}$ receptors are present in the qNSCs state. We also observed that the VCAM $1^{+}$ SVZ NSCs express $M_{1}$ receptors in SVZ NSCs culture (Fig. 7B). 
To study the effect of ACh on the activity of LV qNSCs via binding to the $\mathrm{M}_{1}$ receptors, we first tested whether the pace of generating $\mathrm{DCX}^{+}$cells is affected by adding pirenzepine (a selective M1 receptors antagonist) to cultures of SVZ NSCs treated with carbachol. Adding pirenzepine along with carbachol 2 days or 3 days after differentiation resulted in a significant decrease in produced $\mathrm{DCX}^{+}$cells compared to samples treated with carbachol only $(59.63 \pm 8.9 \%)$ $(19.4 \pm 6.8 \%)$ (Extended Fig. 8A and B). Two days post incubation in the differentiation media, we detected a significant decrease in the number of $\mathrm{DCX}^{+}$cells in samples treated with pirenzepine compared to carbachol-only samples $(45.9 \pm 10.1 \%)$ (Extended Fig. 8C and D). There was no significant difference in the number of $\mathrm{GFAP}^{+}$cells. This indicate that activating of $\mathrm{M}_{1}$ receptors has no impact on the number of $\mathrm{GFAP}^{+} \mathrm{NSCs}$, but increased the number of generated neuroblast (Extended Fig. 8C and E).

To detect the effect of $\mathrm{M}_{1}$ receptors activation on qNSCs, we first prepared SVZ NSCs culture treated with pirenzepine and carbachol for one day in the proliferation media. We noticed a significant decrease in the quantity of pEGFR (PhosphoY1068) protein in pirenzepine accompanied with carbachol-treated samples compared to samples treated with carbachol only $(69.8 \pm 19.2 \%)$ (Fig. 7C and D). This suggested that adding pirenzepine to carbachol treated samples decreases the number of activated $\mathrm{pEGFR}^{+} \mathrm{NSCs}^{+}$cells, which supports that $\mathrm{M}_{1}$ receptors are expressed during the quiescent state of LV NSCs. We also added pirenzepine simultaneously with carbachol and examined the effect on the number of $\mathrm{Ki}^{+} 7^{+}$cells and $\mathrm{EdU} \mathrm{Ki}^{+} / \mathrm{Ki}^{+}$cell ratio. Although no significant differences on the number of $\mathrm{Ki}^{+}$cells were noticed (Fig. 7E and F), the ratio of $\mathrm{EdU}^{+} / \mathrm{Ki}^{+} 7^{+}$cells significantly decreased in samples incubated with carbachol and pirenzepine compared to samples treated with carbachol only (74.6.8 $\pm 5.6 \%$ ) (Fig. 7E and G). These results strongly suggest that the $\mathrm{M}_{1}$ receptor selective antagonist (pirenzepine) prevents carbachol-induced activation of qNSCs.

To further confirm the effect of $\mathrm{M}_{1}$ receptor activation on qNSCs, we studied the effect of adding pirenzepine to samples of SVZ NSCs incubated with carbachol on the quantity of pEGFR (PhosphoY1068) protein during the initial phase of differentiation (Fig. 7H). The SVZ NSCs culture were treated once at beginning of the differentiation stage with either carbachol or carbachol along with pirenzepine. The samples which were incubated with carbachol accompanied with pirenzepine and collected after $12 \mathrm{hrs}$ have shown a significant decrease in the quantity of 
pEGFR (PhosphoY1068) protein (23.7 $\pm 7.4 \%$ ) (Fig. 7H and J). However, the samples that were collected after 24 and 36 hrs have did not show any significant changes in the quantity of pEGFR (PhosphoY1068) protein (Fig. 7I and J). Using the same experimental conditions, we stained treated samples against pEGFR (PhosphoY1068) antibody which were incubated in the differentiation media for one day. The treated samples with pirenzepine revealed a significant decrease in the number of pEGFR ${ }^{+} \mathrm{NSCs}$ compared with carbachol-only samples $(57.1 \pm 21.2 \%)$ (Extended Fig. 8F and G)..

Afterwards, we studied the effect of adding pirenzepine to carbachol-treated samples on the number of $\mathrm{Ki} 67^{+}$cells and $\mathrm{EdU}^{+} / \mathrm{Ki} 67^{+}$cell ratio in the early differentiation stage of SVZ NSCs culture. Although no difference in the number of $\mathrm{Ki} 67^{+}$cells was found, a significant decrease in $\mathrm{EdU}^{+} / \mathrm{Ki} 67^{+}$cells ratio was observed in samples incubated with pirenzepine along with carbachol compared to carbachol-treated samples $(34.5 \pm 6.1 \%)$ (Fig. $7 \mathbf{K}$ and $\mathbf{L})$. This reduction in the number of newly proliferative cells by blocking $\mathrm{M}_{1}$ receptors confirms that $\mathrm{ACh}$ initiates the activation of qNSCs by binding to $\mathrm{M}_{1}$ receptors (Fig. $7 \mathbf{M}$ ).

\section{Discussion}

We identified a novel circuit controlling postnatal/adult neurogenesis in the SVZ niche. Using a Rabies tracing strategy, we determined the source of synaptic inputs to the subep-ChAT ${ }^{+}$ neurons, which are known to direct LV NSC proliferation ${ }^{26}$. Our results showed that subep$\mathrm{ChAT}^{+}$neurons have a different pattern of neural connectivity compared other cholinergic neurons, such as those in the striatum: they are not directly connected with the adjacent striatal $\mathrm{ChAT}^{+}$neurons, or with each other ${ }^{36}$. In particular, we found that glutamatergic inputs from a specific $\mathrm{CR}^{+}$neuronal population in the anterior cingulate cortex that projects directly to subep$\mathrm{ChAT}^{+}$neurons. This is the first identification of a distal cortical input that directly drives subep$\mathrm{ChAT}^{+}$neurons and regulate $\mathrm{ACh}$ release in the $\mathrm{SVZ}$ niche. In addition, we also identified a small population of local $\mathrm{CR}^{+}$GABAergic interneurons that inhibit subep-ChAT ${ }^{+}$neurons. Previously, GABA was shown to be involved in various functions in the SVZ niche such as enhancing neuroblast maturation ${ }^{37}$, and preserving the postnatal/adult NSCs by inhibiting their proliferation and differentiation, but the source of GABA was largely unclear ${ }^{22,38}$. We demonstrated GABAergic $\mathrm{CR}^{+}$interneurons are a local source of GABA (Fig. 2G). 
Together excitatory and inhibitory synaptic inputs can shape the output of subep-ChAT ${ }^{+}$ neurons and thus regulate the release of ACh. In turn, we demonstrated that ACh acts on M1 muscarinic receptors to promote the proliferation of postnatal/adult LV NSCs. In vivo optogenetics and chemogenetic stimulations of $\mathrm{Cg} 1 \mathrm{VGlut}^{+} / \mathrm{CR}^{+}$neurons were sufficient to increase LV NSCs proliferation and SVZ neurogenesis in the wall of the lateral ventricle (Figure 5, Extended Figure 5).

Both postnatal and adult LV NSCs proliferation and SVZ neurogenesis are known to be modulated by neural activity ${ }^{19}$. Recent work on differential gene expression in LV NSCs in quiescent and activated states suggests possible mechanisms for the activation of LV qNSCs ${ }^{39,40}$. In in vitro studies, quiescent-NSCs and activated-NSCs in the postnatal/adult SVZ are known to be interconvertible ${ }^{4}$. While both populations express GFAP, the expression of Notch2 and EGFRs genes are limited to qNSCs and aNSCs, respectively ${ }^{4,41-44}$. Thus, we can use the expression level of GFAP and EGFRs to quantify the relative number of aNSCs.

We found that carbachol during the proliferation stage led to downregulation of Notch2 and upregulation of EGFR genes. As EGFRs are significantly upregulated in the carbachol-treated SVZ NSCs, EGF initiates NSCs proliferation. Within the aNSCs population, fewer $\mathrm{EGFR}^{+} \mathrm{NSCs}$ are positive for $\mathrm{Ki} 67$ gene compared with $\mathrm{EGFR}^{+}$TAC cells ${ }^{39,45}$. These findings confirm the role of ACh in converting qNSCs to aNSCs.

So far no neural pathway has been identified to directly modulate LV NSCs in the quiescent state. Our results demonstrate that $\mathrm{ACh}$ acts on $\mathrm{M}_{1}$ receptors on the LV NSCs during the quiescent state. The effect of $\mathrm{M}_{1}$ receptor activation on non-excitable cells like qNSCs is poorly studied. $\mathrm{M}_{1}$ receptor activates various signaling pathways within the cell, including phospholipase $\mathrm{C}$ (PLC)-mediated formation of inositol 1,4,5-trisphosphate (IP3), which causes $\mathrm{Ca}^{2+}$ release from the endoplasmic reticulum (ER) through binding to IP3 receptors (IP3Rs) ${ }^{46,47}$. Intracellular calcium is known to regulate cell proliferation ${ }^{48}$. Future research may focus on the location of $\mathrm{M}_{1}$ receptors and downstream intracellular signaling within qNSCs.

Taken together, our results reveal for the first time a defined neural circuit that allows direct cortical regulation oof SVZ neurogenesis. In humans, there is evidence for active neurogenesis at the wall of the lateral ventricles that generate migratory neuroblasts for up to two years after birth ${ }^{49}$, but the circuit mechanism is unknown. The analogous process in rodents may shed light on 
how the neurogenesis during early brain development is influenced by neural activity and synaptic inputs. At present, how frontal cortical inputs to the SVZ are related to environmental factors and behavior remains unclear, but our results promise to bridge neural activity and neurogenesis, and open up a new avenue of research into cortical activity-dependent SVZ neurogenesis in adult animals.

\section{Methods}

\section{Animals}

All experiments were approved by the Institutional Animal Care and Use Committee at Duke University. Mice were group housed on a standard $12 \mathrm{~h}$ light/dark cycle (lights on at 7 a.m.) with a controlled average ambient temperature of $21^{\circ} \mathrm{C}$ and $45 \%$ humidity. The following mouse lines were purchased from JAX: C57BL/6J (000664); Vglut1-Cre (023527); ChAT-eGFP (007902)::ChR2(RCL-hChR2(H134R)/tdT)-D (012567); ChAT-Cre (006410); Cr-Cre (010774); Vgat-Cre (016962); Cb-Cre (028532); SST-Cre (013044); PV-Cre (017320); Ai9(RCL-tdT) (007905). We generated the R26R-FLEX-TVA-2A-RabiesG-2A-tdTomato-FLEX (R26F-RTT) mouse line for rabies monosynaptic circuit tracing: mice (Fig. 1F).

\section{Rabies viral retrograde tracing}

An intraventricular approach via a Cre-dependent viral strategy was employed to avoid labeling striatal $\mathrm{ChAT}^{+}$neurons. We used ChAT-Cre mice to express TVA, Rabies-G protein, and tdTomato in subep-ChAT ${ }^{+}$neurons after the first viral infection. The infected subep-ChAT ${ }^{+}$ neurons were later targeted with the second EnvA G-deleted rabies virus to enable efficient monosynaptic tracing. It is extremely difficult to infect the same subep-ChAT ${ }^{+}$neurons via two separate viral injections. To overcome this obstacle, we successfully generated and validated $R 26 R-F L E X$ TVA-2A-RabiesG-2A-tdTomato-FLEX (R26F-RTT) mice (Fig. 1F and G, and Extended Fig. 2). These mice allow for monosynaptic tracing of Cre-targeted neurons via a single EnvA G-deleted Rabies-eGFP (Salk, USA) or rVSV(RABV-G)-eGFP (Salk, USA) viral injection. 


\section{Stereotaxic injections}

Stereotaxic injections were performed as mice were kept deeply anesthetized in a stereotaxic frame (David Kopf Instruments) with isoflurane. For circuit tracing, rabies virus, 300 nL of (EnvA G-deleted Rabies-eGFP or rVSV(RABV-G)-eGFP (Salk, USA)) virus were injected into the striatum or the lateral ventricles of P30 ChAT-Cre:: R26F-RTT mice. Viruses were infused slowly over $10 \mathrm{~min}$ into the striatum as the following coordinates relative to Bregma (AP: $+1, \mathrm{ML}$ $\pm 2.0, \mathrm{DV}: 2.2$ from brain surface) or lateral ventricle (AP: $+0.8, \mathrm{ML} \pm 0.65, \mathrm{DV}: 2.1$ from brain surface) using a microdriver with a $10 \mu \mathrm{L}$ Hamilton syringe. For electrophysiological testing of $\mathrm{Cg} 1$ inputs to the subep-ChAT ${ }^{+}$neurons (optogenetic-light stimulation), adeno-associated viral viruses were used for Cre-dependent expression of the excitatory channelrhodopsin. AAVCaMKII-hChR2(E123A)-mCherry (addgene \#35506) (300nl) virus or pAAV-Ef1a-DIO hChR2(E123T/T159C)-EYFP (addgene \#35509) (300nl) virus were injected into C57BL/6J(P30) or ChAT-Cre:: R26F-RTT (P30) mice, respectively. Also, pAAV-Efla-DIO hChR2(E123T/T159C)-mCherry (addgene \#35510) (300nl) virus was injected into Vglut1-Cre:: ChAT-eGFP (P30) and Cr-Cre:: ChAT-eGFP (P30) mice. Viruses were infused slowly over 10 min into the Cg1 using the following coordinates relative to Bregma (AP: $+0.9, \mathrm{ML} \pm 0.25, \mathrm{DV}$ : 0.3 from brain surface). For in vivo optogenetic testing of cortical inputs to the subep-ChAT ${ }^{+}$ neurons, pAAV-Ef1a-DIO hChR2(E123T/T159C)-mCherry (300nl) virus was injected into $\mathrm{Cr}$ Cre (P30) mice. Viruses were infused slowly over 10 min into the Cg1 (AP: +0.9, ML \pm 0.25, DV: 0.3 from brain surface). pAAVrg-hSyn-DIO hM3D(Gq)-mCherry (addgene \#44361-AAVrg) (300 nl) was injected into the lateral ventricle (AP: $+0.8, \mathrm{ML} \pm 0.65, \mathrm{DV}: 2.1$ from brain surface). For in vivo chemogenetics testing of $\mathrm{Cg} 1$ inputs to the subep-ChAT ${ }^{+}$neurons, pAAV-hSyn-DIOhM3D(Gq)-mCherry (addgene \#44361) (300nl) virus were injected into Cr-Cre (P30) mice. Viruses were infused slowly over $10 \mathrm{~min}$ into the $\mathrm{Cg} 1$ as the following coordinates relative to Bregma (AP: $+0.9, \mathrm{ML} \pm 0.25, \mathrm{DV}: 0.3$ from brain surface). All viruses were infused slowly for over 10 min using a Nanoject (Drummond Scientific) connected to a glass pipette. The injection pipette was left in place for 10 min post-injection before it was retracted.

\section{SVZ wholemount preparation and whole-cell patch-clamp recording}


For electrophysiology experiments both male and female mice (4- to 7-week-old) were used. they were anesthetized with isofluorane, transcardially perfused and then ventricular wall were dissected as whole mounts in ice-cold NMDG artificial cerebrospinal fluid (ACSF; containing $92 \mathrm{mM}$ NMDG, $2.5 \mathrm{mM} \mathrm{KCl}, 1.2 \mathrm{mM} \mathrm{NaH}_{2} \mathrm{PO}_{4}, 30 \mathrm{mM} \mathrm{NaHCO}$, $20 \mathrm{mM}$ HEPES, 2 $\mathrm{mM}$ glucose, $5 \mathrm{mM}$ sodium ascorbate, $2 \mathrm{mM}$ thiourea, $3 \mathrm{mM}$ sodium pyruvate, $10 \mathrm{mM} \mathrm{MgSO}_{4}$, $0.5 \mathrm{mM} \mathrm{CaCl}_{2}$ ), and bubbled with $5 \% \mathrm{CO}_{2} / 95 \% \mathrm{O}_{2}$. Tissues were then bubbled in same solution at $37^{\circ} \mathrm{C}$ for $15 \mathrm{~min}$, transferred to bubbled, modified-HEPES ACSF at $23-25^{\circ} \mathrm{C}(92 \mathrm{mM} \mathrm{NaCl}$, $2.5 \mathrm{mM} \mathrm{KCl}, 1.2 \mathrm{mM} \mathrm{NaH}_{2} \mathrm{PO}_{4}, 30 \mathrm{mM} \mathrm{NaHCO}_{3}, 20 \mathrm{mM}$ HEPES, $2 \mathrm{mM}$ glucose, $5 \mathrm{mM}$ sodium ascorbate, $2 \mathrm{mM}$ thiourea, $3 \mathrm{mM}$ sodium pyruvate, $2 \mathrm{mM} \mathrm{MgSO}_{4}, 2 \mathrm{mM} \mathrm{CaCl}_{2}$ ) for at least 45 min before start experimentation. (For NMDG artificial cerebrospinal fluid and modified-HEPES ACSF solutions, $\mathrm{pH}$ was adjusted to 7.5 and osmolarity to $290 \mathrm{mOsm}$ ). Recordings were performed in submerged chamber, superfused with continuously bubbled ACSF $(125 \mathrm{mM} \mathrm{NaCl}$, $2.5 \mathrm{mM} \mathrm{KCl}, 1.25 \mathrm{mM} \mathrm{NaH}_{2} \mathrm{PO}_{4}, 26 \mathrm{mM} \mathrm{NaHCO}_{3}, 20 \mathrm{mM}$ glucose, $2 \mathrm{mM} \mathrm{CaCl}$, $1.3 \mathrm{mM} \mathrm{MgCl}_{2}$ ) at $2.5-5 \mathrm{ml} / \mathrm{min}$ at $23-25^{\circ} \mathrm{C}$.

Patch electrodes with a resistance of 3-6 M $\Omega$ were pulled from borosilicate glass capillaries using a horizontal puller (P-97, Sutter-instruments). All subep-ChAT ${ }^{+}$neurons used in the electrophysiology recordings were identified by their distinctive morphology from striatal $\mathrm{ChAT}^{+}$neurons, location (within 15-20 $\mu \mathrm{M}$ from surface), their non-spontaneous firing activity, and their depolalrized resting membrane potentials $(\sim-45 \mathrm{mV})$. For measuring EPSCs, the following internal solution was used: $130 \mathrm{mM}$ potassium gluconate, $2 \mathrm{mM} \mathrm{NaCl}, 4 \mathrm{mM} \mathrm{MgCl}$, $20 \mathrm{mM}$ HEPES, $4 \mathrm{mM} \mathrm{Na}$ ATP, $0.4 \mathrm{mM} \mathrm{NaGTP}$ and $0.5 \mathrm{mM}$ EGTA, pH adjusted to 7.2 with KOH. A $40 \mu \mathrm{M}$ picrotoxin ((124-87-8), Millipore Sigma) was added in the bath solution in voltage-clamp mode. For measuring inward IPSCs when holding at - $60 \mathrm{mV}$, we used internal solution containing $\mathrm{K}-\mathrm{Cl}$ (high Chloride) $\mathrm{KCl}$ (135 Mm), HEPES (10 mM), Na2ATP (2mM), $\operatorname{NaGTP}(0.2 \mathrm{mM}), \mathrm{MgCl} 2(2 \mathrm{mM})$, and EGTA $(0.1 \mathrm{mM}), \mathrm{pH}$ adjusted to 7.2 with $\mathrm{KOH}$. To block glutamatergic transmission, $50 \mu \mathrm{M}$ DL-AP5 (NMDA antagonist) ((3693/50), R\&D Systems) and $50 \mu \mathrm{M}$ CNQX (AMPA antagonist) ((0190), Tocris) were added in the bath solution in voltageclamp mode. To block action potentials in the patched cell, the sodium channel blocker QX-314 (10 mM, 552233, Millipore Sigma) was also added. Signals were amplified with Multiclamp 700B (filtered at $10 \mathrm{kHz})$, digitized with Digidata 1440A $(20 \mathrm{kHz})$, and recorded using pClamp 10 
software (Axon). Light-activation of channelrhodopsin was achieved using a 473-nm laser (X-Cite exacte) through a 40x objective (Nikon). Action potentials and evoked EPSCs and IPSCs were analyzed using Neuromatic package (Think Random) in Igor Pro software (WaveMetrics) and AxoGrapth.

For focal (targeted) photostimulation of local $\mathrm{CR}^{+}$-IPSCs from subep-ChAT ${ }^{+}$neurons, we used Polygon 400 Digital Micromirror Device (Mightex; multiwavelength pattered illuminator) to control the temporal dynamics (the size, shape, intensity, and position) of light inputs. The illumination consisted of a $100 \mathrm{~ms}$ light pulse $(470-\mathrm{nm})$ at $50 \%$ intensity in the selected area (50 $\mu \mathrm{m})$. The pulse was triggered using a TTL pulse from the Digidata to synchronize the stimulation with electrophysiology. Photostimulation was performed for one fluorescent subep-CR $\mathrm{CR}^{+}$neuron presents in the field of view resulted in postsynaptic effects in the recorded neuron. One or two cells were recorded per wholemount SVZ. Up to three cells from each animal were recorded.

\section{Immunofluorescence staining and imaging}

Preparation of brain tissue for IHC staining was described previously ${ }^{26}$. We used primary antibodies to GFP (\#GFP-1020, 1:400, AVES lab), Choline Acetyltransferase Antibody (\#AB144P, 1:250, Millipore sigma), tdTomato [16D7] (\#EST203, 1:200, Kerafast), RFP (\#600401-379, 1:250, Rockland), Calretinin (\#ab702, 1:200, Abcam), calretinin (\#6B3, 1:250, Swant), Calretinin (\#MCA-3G9, 1:250, EnCor Biotechnology), RFP (\#ab62341, 1:200, Abcam), Doublecortin (\#AB2253, 1:250, Millipore), Doublecortin (\#4604S, 1:200, Cell Signaling Technology), Ki67 (ab15580, 1:250: Abcam), Ki67 (\#CPCA-Ki67, 1:200, EnCor Biotechnology), beta Actin HRP (\#MA515739HRP (BA3R), 1:5000, Thermo Fisher Scientific), HRP-conjugated Beta Actin (\#HRP-60008, 1:5000, Proteintech), EGFR (phospho Y1068) (\#ab5644, 1:250, Abcam), EGFR (phospho Y1068) [Y38] (\#ab32430, 1:250, Abcam), CD106 (\#550547, 1:100, BD Diagnostic Systems), GFAP (\#GFAP, 1:500, Aves Labs), M1 Muscarinic Receptor (\#AMR-001, Alomone Labs). In brief, for circuit tracing, in vivo optogenetic and in vivo chemogenetics experiments, after experiments mice were deeply anesthetized with isoflurane, perfused transcardially with phosphate buffered saline (PBS), followed by 4\% PFA in PBS. Perfused brains were removed and postfixed overnight at $4^{\circ} \mathrm{C}$ in $4 \%$ PFA. Brains were cut into $50 \mu \mathrm{m}$ coronal sections by Leica VT1000S vibratome or Precisionary Instruments VF-500-0Z vibrating 
microtome. Slices were incubated in a blocking solution containing 5\% donkey serum and TBST for $100 \mathrm{~min}$ at room temperature. Slices were then incubated in room temperature overnight in PBS containing 1\% donkey serum and antibodies. Slices were then washed with PBS and incubated with secondary antibodies, Alexa-594 (1:1000, LifeTech) or Alexa-488 (1:1000, LifeTech) or Alexa-647 (1:1000, LifeTech) for $2 \mathrm{~h}$ at room temperature, before washing with PBS. Sections were counterstained with a 4',6-diamidino-2-phenylindole solution (DAPI; (D9542) Sigma-Aldrich). After washing four times with TBST, the sections were coverslipped with Fluoromount (Sigma) aqueous mounting medium. Images were taken by tile scan imaging using Leica SP8 upright confocal microscope (Zeiss) with $10 \times, 20 \times$ and $40 \times$ objectives under the control of Zen software (Zeiss). All antibodies used were validated as in previous publications ${ }^{26,50}$ or by publications available on vendor website specific to each antibody.

To our knowledge there is no previous report of an excitatory CR-expressing cell population in the cingulate cortex. When we stained the $\mathrm{Cg} 1 \mathrm{CR}^{+}$neurons that project to subep$\mathrm{ChAT}^{+}$neurons using commercially available calretinin antibodies, we did not see any colocalization with $\mathrm{CR}^{+}$neurons in the $\mathrm{Cg} 1$ region. Antibodies have intrinsic limitations in their sensitivity and specificity, which could explain why calretinin antibodies did not detect this small population of $\mathrm{CR}^{+}$neurons. Although the $\mathrm{Cr}$-Cre line was originally designed to target inhibitory neurons in the mouse brain ${ }^{51}$, Cre-activated reporters amplify gene expression levels, and probably provides more sensitive measures to discern endogenous gene expression than immunostaining.

\section{In vivo optogenetic stimulation}

Cannulae targeting the $\mathrm{Cg} 1$ region were implanted (AP: $+0.9, \mathrm{ML} \pm 0.25, \mathrm{DV}: 0.3$ from brain surface) after injecting pAAV-Ef1a-DIO hChR2(E123T/T159C)-mCherry virus as described in Fig. 5a, using implantable mono fiber-optic fiber $(200 \mu \mathrm{m}, 0.22 \mathrm{NA}$, Doric). Protruding ferrule end of cannula was then connected via fiber cord and a rotary coupling joint (Doric) was used to permit free movement. Two to three weeks after viral infection, light-stimulation was delivered by TTL control (Master 8, AMPI) of 473-nm laser (IkeCool), $10 \mathrm{~ms}$ pulses at $10 \mathrm{~Hz}$, lasting $10 \mathrm{~s}$, given once every $1 \mathrm{~min}$. For ChAT, Ki67, DCX and RFP analyses, 50- $\mu \mathrm{m}$ brain coronal sections were cut and collected on Leica VT1000S vibratome or Precisionary Instruments VF-500-0Z vibrating microtome. Coronal sections surrounding the fiber tract were selected for analyses. 


\section{In vivo chemogenetics}

The Clozapine N-oxide (CNO - HB1807) (Hello Bio, Bristol, UK) was Dissolved in 0.9\% saline and stored at $-20{ }^{\circ} \mathrm{C}$ until used as shown in (Extended Fig. 5). All the injections were given intraperitoneally, at a volume of $1 \mathrm{mg} / \mathrm{kg}$ daily for 5 days. For DCX analysis, $50-\mu \mathrm{m}$ brain coronal sections were cut and collected on Leica VT1000S vibratome or Precisionary Instruments VF-500$0 \mathrm{Z}$ vibrating microtome. Coronal sections of slices at AP: $+0.75-1 \mathrm{~mm}$ relative to Bregma were selected for analyses, comparing littermates with activation versus no-activation (Control).

\section{SVZ NSCs culture}

The SVZ NSCs culture were performed as described in our previous publication and others 26,52. In brief, SVZ wholemount from P12 C57BL/6J mice were dissected and placed in DMEM/F12 (DF) containing 100 units $/ \mathrm{ml}$ penicillin, $100 \mu \mathrm{g} / \mathrm{ml}$ streptomycin, and $250 \mathrm{ng} / \mathrm{ml}$ amphotericin $\mathrm{B}$ (collectively, abx). The tissues were pooled and incubated in $0.005 \% \operatorname{trypsin}\left(15 \mathrm{~min}, 37^{\circ} \mathrm{C}, \mathrm{pH}\right.$ 7.3) and placed overnight in uncoated T75 plastic tissue-culture dishes in N5 medium (DF containing N2 supplements, $35 \mu \mathrm{g} / \mathrm{ml}$ bovine pituitary extract, abx, 5\% FCS (HyClone), and 40 $\mathrm{ng} / \mathrm{ml}$ EGF and basic FGF). Unattached cells were collected and then plated again onto uncoated plastic dishes. These cells were proliferated to confluency in N5 media. EGF and FGF (20 ng each) were added every other day. Cells were passaged two or more times by using $0.005 \%$ trypsin and N5 media, with $20 \mathrm{ng} / \mathrm{ml}$ EGF/FGF supplementation every other day. The cells were proliferated to $90-100 \%$ confluency and were induced to differentiate by removing growth factors and serum from the culture media (N6 media; DF containing N2 supplements, $35 \mu \mathrm{g} / \mathrm{ml}$ bovine pituitary extract, and abx). More details of various treatment conditions for different experiments were included in figure legends.

\section{SDS-PAGE and immunoblotting}

Protein extracts were prepared as previously described ${ }^{26}$, and resolved by electrophoresis through SDS-PAGE and transferred onto nitrocellulose membranes. Antibodies were diluted in PBS containing $0.2 \%$ Triton X-100 (vol/vol) and 4\% non-fat dry milk (wt/vol), followed by 
overnight incubation at $4{ }^{\circ} \mathrm{C}$. Detection was accomplished through secondary antibodies conjugated to horseradish peroxidase (\#111-036-003, 115-036-003, 112-005-006, 106-006-003, 1:5 mL, Jackson ImmunoResearch) and treated with enhanced chemiluminescence (\#1705061; Bio-Rad Laboratories). Intensity of DCX or pEGFR bands were quantified with ImageJ and their values were then normalized vs. $\beta$-actin bands. The final quantification values are the ratio of net band to net loading control.

\section{EdU staining}

The SVZ NSCs culture were plated on glass coverslips coated in DMEM/F-12. 5-ethynyl2'-deoxyuridine (EdU) staining was conducted using Click-iT ${ }^{\mathrm{TM}}$ EdU Cell Proliferation Imaging Kit (ThermoFisher, USA) according to the manufacture's protocol. EdU was added to the culture medium at a concentration of $10 \mu \mathrm{M}$ for 15 minutes. After labeling, cells were washed three times with PBS. Cells were then permeabilized in 0.5\% TBST for 30 seconds and then fixed in $10 \%$ formaldehyde for 15 minutes. Cells were then rinsed twice with PBS and then incubated for 25 minutes in Click-i ${ }^{\mathrm{TM}}$ reaction cocktail contacting Click-i $\mathrm{T}^{\mathrm{TM}}$ reaction buffer, $\mathrm{CuSO}_{4}$, Alexa fluor 647 Azide, and reaction buffer additive while protected from light. After staining, the cells on coverslips were washed three times with PBS plus 0.5\% Triton X-100, 5 minutes each. Cells were then counterstained with DAPI, mounted in vectashield mounting media (vector laboratories Inc, Burlingame, CA) and imaged by using Leica SP8 upright confocal microscope (Zeiss) as above. All steps were carried out at room temperature.

\section{Total RNA extraction, cDNA synthesis, and Quantitative PCR}

The SVZ NSCs culture in a proliferation media of control and treated samples were extracted using RNeasy plus Mini Kit (QIAGEN, USA), according to the manufacture's protocol. Then, the first-strand cDNAs were synthesized on a SimpliAmp ${ }^{\mathrm{TM}}$ thermocycler (ThermoFisher Scientific) using SuperScript ${ }^{\mathrm{TM}}$ VILO $^{\mathrm{TM}}$ cDNA Synthesis Kit (Invitrogen, USA), according to the manufacture's protocol. 
For qPCR experiments, Power SYBR Green Master Mix (Applied Biosystems, USA) were used, and the qPCR experiments were conducted using the StepOnePlus Real-Time PCR System running StepOne Design and Analysis Software (Applied Biosystems). All reaction conditions and protocols were performed as recommended by the manufacturer. Copies/reaction were determined by absolute quantification.

Primers for ChRM1 and $\beta$-Actin genes:

ChRM1 F 5' CAG TCC CAA CAT CAC CGT CTT 3'

ChRM1 R 5' GAG AAC GAA GGA AAC CAA CCA C 3'

$\beta$-Actin F 5' GGC TGT ATT CCC CTC CAT CG 3'

$\beta$-Actin R 5' CCA GTT GGT AAC AAT GCC ATG 3'

\section{Poly-A RNA Sequencing (RNASeq)}

For total RNA extraction of the SVZ NSCs culture samples, SVZ NSCs cultures in proliferation media with carbachol was started in half of samples for 24 hours only. Then the total cellular RNA was extracted using RNeasy® Plus Mini Kit (Qiagen) and sent to LC Sciences company, wherethe RNA integrity of total RNA samples was checked using an Agilent Technologies 2100 Bioanalyzer. The poly-A containing mRNA molecules were purified using poly-T oligo attached magnetic beads and using two rounds of purification. After purification the poly-A RNA was fragmented using divalent cation containing buffer and elevated temperature. The first strand cDNA was obtained by reverse transcription using reverse transcriptase and a random primer. The second strand cDNA was synthesized by the process of removing the RNA template, synthesizing a replacement strand and dUTP method for strandspecificity. After the 3'-end adenylation and 5'-end phosphorylation, the blunt-ended cDNA adapters were ligated to the ends of the ds-cDNA. PCR amplification was performed to enrich the ligated cDNA and generate the library for sequencing. Quality control analysis and quantification of the DNA library were performed using agarose gel or Agilent Technologies 2100 Bioanalyzer High Sensitivity DNA Chip and KAPA Library Quantification Kits. Paired-end sequencing was performed on Illumina's NovaSeq sequencing system. 


\section{Quantification and Statistical Analysis}

All data are expressed as mean \pm SEM and all statistical analyses were performed using GraphPad Prism (version 8). Unpaired t-test was used for analysis of in vivo optogenetics stimulation, in vivo chemogenetics stimulation, and in vitro SVZ NSCs culture modulation studies analysis. $\mathrm{p}<0.05$ was considered statistically significant.

\section{Acknowledgements}

We thank Scott Soderling and Shawn Je for helpful comments on the manuscript, Transgenic and Knockout Mouse Facility at Duke University for assistance with generation of R26R-FLEX-TVA2A-RabiesG-2A-tdTomato-FLEX mice, LC Sciences company for helping with Poly-A RNA sequencing experiment and analysis, E. Adlaf, J. Erb, B. Asrican, D. Fromme, P. Paez-Gonzalez, K. Abdi, G. Neves, S. Ramamoorthy, J. David and K. Woldemichael for project assistance. This work was supported by NIH grant R01MH105416.

\section{Contributions}

M.M.N., C.T.K. and H.H.Y. conceived the project and participated in research design. M.M.N. performed all experiments and analyzed data. R.R.K. helped with cell culture experiments. M.M.N. and H.H.Y wrote the paper.

\section{Reference}

1 Lim, D. A. \& Alvarez-Buylla, A. The Adult Ventricular-Subventricular Zone (V-SVZ) and Olfactory Bulb (OB) Neurogenesis. Cold Spring Harb Perspect Biol 8, doi:10.1101/cshperspect.a018820 (2016).

2 Ihrie, R. A. \& Alvarez-Buylla, A. Lake-front property: a unique germinal niche by the lateral ventricles of the adult brain. Neuron 70, 674-686, doi:10.1016/j.neuron.2011.05.004 (2011).

3 Lazarini, F. \& Lledo, P. M. Is adult neurogenesis essential for olfaction? Trends Neurosci 34, 2030, doi:10.1016/j.tins.2010.09.006 (2011). 
4 Codega, P. et al. Prospective identification and purification of quiescent adult neural stem cells from their in vivo niche. Neuron 82, 545-559, doi:10.1016/j.neuron.2014.02.039 (2014).

5 Mich, J. K. et al. Prospective identification of functionally distinct stem cells and neurosphereinitiating cells in adult mouse forebrain. Elife 3, e02669, doi:10.7554/eLife.02669 (2014). Doetsch, F., Caille, I., Lim, D. A., Garcia-Verdugo, J. M. \& Alvarez-Buylla, A. Subventricular zone astrocytes are neural stem cells in the adult mammalian brain. Cell 97, 703-716, doi:10.1016/s0092-8674(00)80783-7 (1999).

7 Obernier, K. et al. Adult Neurogenesis Is Sustained by Symmetric Self-Renewal and Differentiation. Cell Stem Cell 22, 221-234 e228, doi:10.1016/j.stem.2018.01.003 (2018). Lois, C., Garcia-Verdugo, J. M. \& Alvarez-Buylla, A. Chain migration of neuronal precursors. Science 271, 978-981, doi:10.1126/science.271.5251.978 (1996).

9 Ponti, G., Obernier, K. \& Alvarez-Buylla, A. Lineage progression from stem cells to new neurons in the adult brain ventricular-subventricular zone. Cell Cycle 12, 1649-1650, doi:10.4161/cc.24984 (2013).

10 Lois, C. \& Alvarez-Buylla, A. Long-distance neuronal migration in the adult mammalian brain. Science 264, 1145-1148, doi:10.1126/science.8178174 (1994).

11 Luskin, M. B. Restricted proliferation and migration of postnatally generated neurons derived from the forebrain subventricular zone. Neuron 11, 173-189, doi:10.1016/0896-6273(93)90281u (1993).

12 Petreanu, L. \& Alvarez-Buylla, A. Maturation and death of adult-born olfactory bulb granule neurons: role of olfaction. J Neurosci 22, 6106-6113, doi:20026588 (2002).

13 Imayoshi, I. et al. Roles of continuous neurogenesis in the structural and functional integrity of the adult forebrain. Nat Neurosci 11, 1153-1161, doi:10.1038/nn.2185 (2008).

14 Livneh, Y., Adam, Y. \& Mizrahi, A. Odor processing by adult-born neurons. Neuron 81, 10971110, doi:10.1016/j.neuron.2014.01.007 (2014).

15 Sakamoto, M. et al. Continuous postnatal neurogenesis contributes to formation of the olfactory bulb neural circuits and flexible olfactory associative learning. J Neurosci 34, 5788-5799, doi:10.1523/JNEUROSCI.0674-14.2014 (2014).

16 Mak, G. K. \& Weiss, S. Paternal recognition of adult offspring mediated by newly generated CNS neurons. Nat Neurosci 13, 753-758, doi:10.1038/nn.2550 (2010).

17 Sakamoto, M., Kageyama, R. \& Imayoshi, I. The functional significance of newly born neurons integrated into olfactory bulb circuits. Front Neurosci 8, 121, doi:10.3389/fnins.2014.00121 (2014).

18 Jones, K. S. \& Connor, B. Intrinsic regulation of adult subventricular zone neural progenitor cells and the effect of brain injury. Am J Stem Cells 1, 48-58 (2012).

19 Obernier, K. \& Alvarez-Buylla, A. Neural stem cells: origin, heterogeneity and regulation in the adult mammalian brain. Development 146, doi:10.1242/dev.156059 (2019).

20 Bovetti, S., Gribaudo, S., Puche, A. C., De Marchis, S. \& Fasolo, A. From progenitors to integrated neurons: role of neurotransmitters in adult olfactory neurogenesis. J Chem Neuroanat 42, 304316, doi:10.1016/j.jchemneu.2011.05.006 (2011).

21 Young, S. Z., Taylor, M. M. \& Bordey, A. Neurotransmitters couple brain activity to subventricular zone neurogenesis. Eur J Neurosci 33, 1123-1132, doi:10.1111/j.14609568.2011.07611.x (2011).

22 Liu, X., Wang, Q., Haydar, T. F. \& Bordey, A. Nonsynaptic GABA signaling in postnatal subventricular zone controls proliferation of GFAP-expressing progenitors. Nat Neurosci 8, 11791187, doi:10.1038/nn1522 (2005).

23 Hoglinger, G. U. et al. Dopamine depletion impairs precursor cell proliferation in Parkinson disease. Nat Neurosci 7, 726-735, doi:10.1038/nn1265 (2004). 
24 Brezun, J. M. \& Daszuta, A. Depletion in serotonin decreases neurogenesis in the dentate gyrus and the subventricular zone of adult rats. Neuroscience 89, 999-1002, doi:10.1016/s03064522(98)00693-9 (1999).

25 Banasr, M., Hery, M., Printemps, R. \& Daszuta, A. Serotonin-induced increases in adult cell proliferation and neurogenesis are mediated through different and common 5-HT receptor subtypes in the dentate gyrus and the subventricular zone. Neuropsychopharmacology 29, 450460, doi:10.1038/sj.npp.1300320 (2004).

26 Paez-Gonzalez, P., Asrican, B., Rodriguez, E. \& Kuo, C. T. Identification of distinct ChAT(+) neurons and activity-dependent control of postnatal SVZ neurogenesis. Nat Neurosci 17, 934942, doi:10.1038/nn.3734 (2014).

27 Oldenburg, I. A. \& Ding, J. B. Cholinergic modulation of synaptic integration and dendritic excitability in the striatum. Current opinion in neurobiology 21, 425-432 (2011).

28 Madisen, L. et al. A toolbox of Cre-dependent optogenetic transgenic mice for light-induced activation and silencing. Nature neuroscience 15, 793-802, doi:nn.3078 [pii]

10.1038/nn.3078 (2012).

29 Lim, S. A., Kang, U. J. \& McGehee, D. S. Striatal cholinergic interneuron regulation and circuit effects. Front Synaptic Neurosci 6, 22, doi:10.3389/fnsyn.2014.00022 (2014).

30 Beier, K. T., Saunders, A. B., Oldenburg, I. A., Sabatini, B. L. \& Cepko, C. L. Vesicular stomatitis virus with the rabies virus glycoprotein directs retrograde transsynaptic transport among neurons in vivo. Front Neural Circuits 7, 11, doi:10.3389/fncir.2013.00011 (2013).

31 Morshead, C. M. et al. Neural stem cells in the adult mammalian forebrain: a relatively quiescent subpopulation of subependymal cells. Neuron 13, 1071-1082, doi:10.1016/0896-6273(94)900469 (1994).

32 Morita, M., Kozuka, N., Itofusa, R., Yukawa, M. \& Kudo, Y. Autocrine activation of EGF receptor promotes oscillation of glutamate-induced calcium increase in astrocytes cultured in rat cerebral cortex. J Neurochem 95, 871-879, doi:10.1111/j.1471-4159.2005.03430.x (2005).

33 Chaker, Z., Codega, P. \& Doetsch, F. A mosaic world: puzzles revealed by adult neural stem cell heterogeneity. Wiley Interdiscip Rev Dev Biol 5, 640-658, doi:10.1002/wdev.248 (2016).

34 Cochard, L. M. et al. Manipulation of EGFR-Induced Signaling for the Recruitment of Quiescent Neural Stem Cells in the Adult Mouse Forebrain. Front Neurosci 15, 621076, doi:10.3389/fnins.2021.621076 (2021).

35 Marques-Torrejon, M. A. et al. LRIG1 is a gatekeeper to exit from quiescence in adult neural stem cells. Nat Commun 12, 2594, doi:10.1038/s41467-021-22813-w (2021).

36 Schafer, M. K., Eiden, L. E. \& Weihe, E. Cholinergic neurons and terminal fields revealed by immunohistochemistry for the vesicular acetylcholine transporter. I. Central nervous system. Neuroscience 84, 331-359, doi:10.1016/s0306-4522(97)00516-2 (1998).

37 Young, S. Z. et al. NKCC1 knockdown decreases neuron production through GABA(A)-regulated neural progenitor proliferation and delays dendrite development. J Neurosci 32, 13630-13638, doi:10.1523/JNEUROSCI.2864-12.2012 (2012).

38 Alfonso, J., Le Magueresse, C., Zuccotti, A., Khodosevich, K. \& Monyer, H. Diazepam binding inhibitor promotes progenitor proliferation in the postnatal SVZ by reducing GABA signaling. Cell Stem Cell 10, 76-87, doi:10.1016/j.stem.2011.11.011 (2012).

39 Dulken, B. W., Leeman, D. S., Boutet, S. C., Hebestreit, K. \& Brunet, A. Single-Cell Transcriptomic Analysis Defines Heterogeneity and Transcriptional Dynamics in the Adult Neural Stem Cell Lineage. Cell Rep 18, 777-790, doi:10.1016/j.celrep.2016.12.060 (2017). 
40 Zywitza, V., Misios, A., Bunatyan, L., Willnow, T. E. \& Rajewsky, N. Single-Cell Transcriptomics Characterizes Cell Types in the Subventricular Zone and Uncovers Molecular Defects Impairing Adult Neurogenesis. Cell Rep 25, 2457-2469 e2458, doi:10.1016/j.celrep.2018.11.003 (2018).

41 Beckervordersandforth, R. et al. In vivo fate mapping and expression analysis reveals molecular hallmarks of prospectively isolated adult neural stem cells. Cell Stem Cell 7, 744-758, doi:10.1016/j.stem.2010.11.017 (2010).

42 Capela, A. \& Temple, S. LeX/ssea-1 is expressed by adult mouse CNS stem cells, identifying them as nonependymal. Neuron 35, 865-875, doi:10.1016/s0896-6273(02)00835-8 (2002).

43 Khatri, P. et al. Proliferation and cilia dynamics in neural stem cells prospectively isolated from the SEZ. Sci Rep 4, 3803, doi:10.1038/srep03803 (2014).

44 Llorens-Bobadilla, E. et al. Single-Cell Transcriptomics Reveals a Population of Dormant Neural Stem Cells that Become Activated upon Brain Injury. Cell Stem Cell 17, 329-340, doi:10.1016/j.stem.2015.07.002 (2015).

45 Aguirre, A., Rubio, M. E. \& Gallo, V. Notch and EGFR pathway interaction regulates neural stem cell number and self-renewal. Nature 467, 323-327, doi:10.1038/nature09347 (2010).

46 Felder, C. C. Muscarinic acetylcholine receptors: signal transduction through multiple effectors. FASEB J 9, 619-625 (1995).

47 Caulfield, M. P. Muscarinic receptors--characterization, coupling and function. Pharmacol Ther 58, 319-379, doi:10.1016/0163-7258(93)90027-b (1993).

48 Patergnani, S. et al. Various Aspects of Calcium Signaling in the Regulation of Apoptosis, Autophagy, Cell Proliferation, and Cancer. Int J Mol Sci 21, doi:10.3390/ijms21218323 (2020).

49 Sanai, N. et al. Corridors of migrating neurons in the human brain and their decline during infancy. Nature 478, 382-386, doi:10.1038/nature10487 (2011).

50 Benner, E. J. et al. Protective astrogenesis from the SVZ niche after injury is controlled by Notch modulator Thbs4. Nature 497, 369-373, doi:10.1038/nature12069 (2013).

51 Taniguchi, H. et al. A resource of Cre driver lines for genetic targeting of GABAergic neurons in cerebral cortex. Neuron 71, 995-1013, doi:10.1016/j.neuron.2011.07.026 (2011).

52 Scheffler, B. et al. Phenotypic and functional characterization of adult brain neuropoiesis. Proc Natl Acad Sci U S A 102, 9353-9358, doi:10.1073/pnas.0503965102 (2005). 

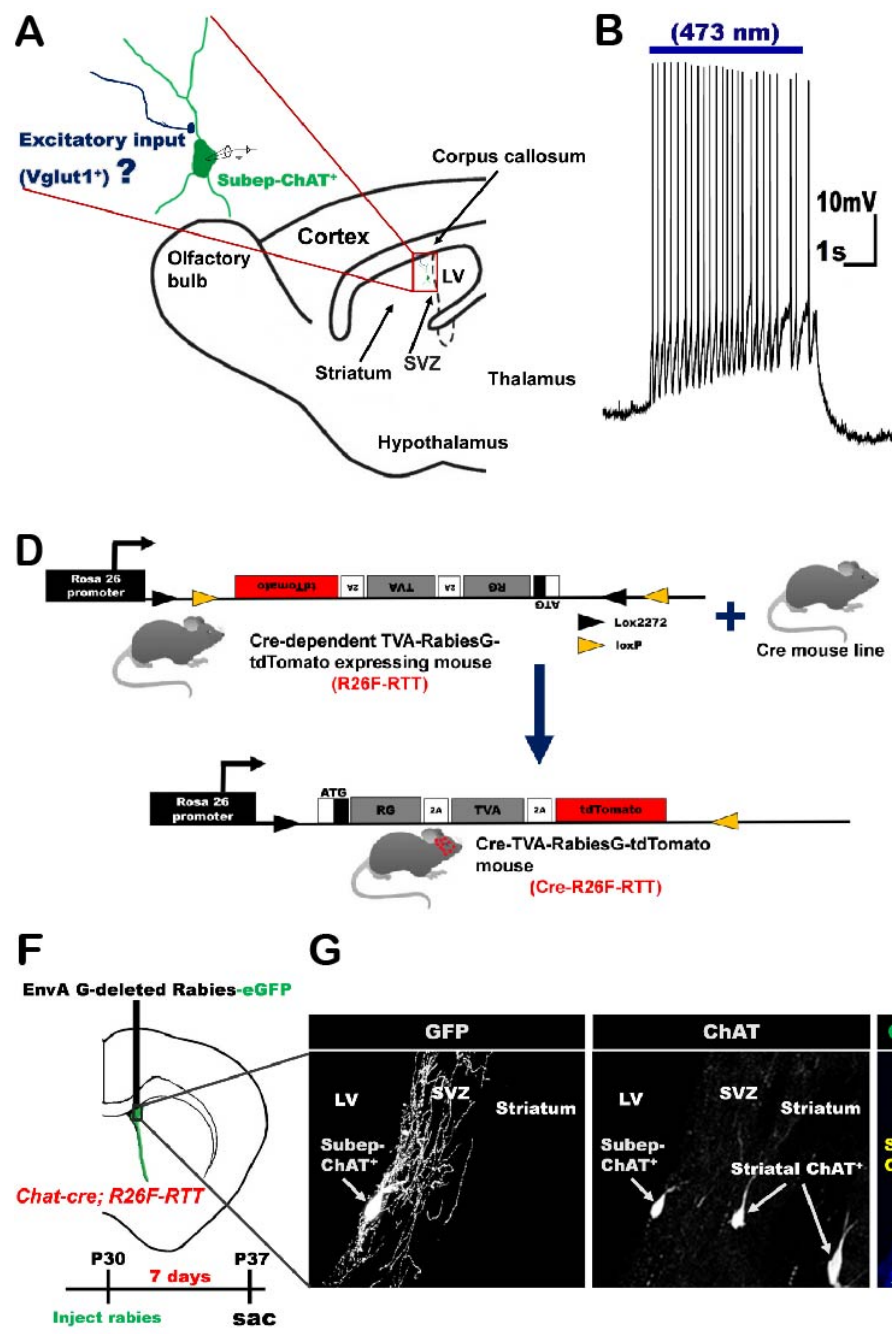
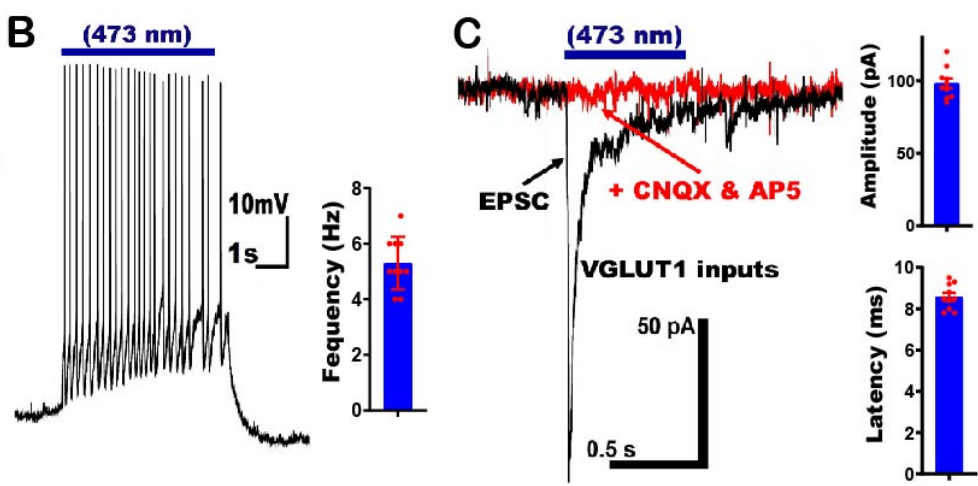

E

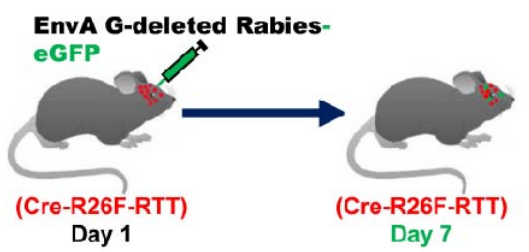

$\mathbf{H}_{\mathrm{s}}$

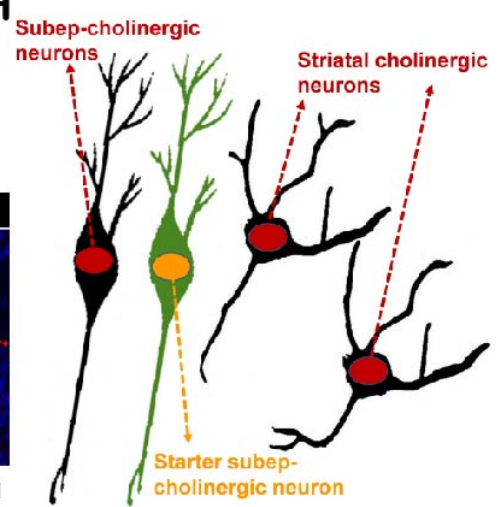

Figure 1. Glutamatergic inputs to subep-ChAT ${ }^{+}$neurons A. Schema showing electrophysiological recording of presynaptic excitatory inputs to the subep-ChAT ${ }^{+}$neuron from P40 VGlut-Cre::ChAT-eGFP ::ChR2 mice. B. Whole cell current clamp recoding of evoked action potential (APs) from subep-ChAT ${ }^{+}$neurons upon blue $(473 \mathrm{~nm})$ light stimulation for $5 \mathrm{~s}$ (Left). Average frequency (Right). $\mathrm{P}<0.0001, t_{9}=17.7, n=10$. C. EPSCs were obtained in whole-cell voltage-clamp recordings from subep-ChAT ${ }^{+}$neurons after photostimulation for $500 \mathrm{~m}$; it is blocked by AMPA and NMDA receptors antagonists; CNQX and AP-5, respectively (Left). Average evoked EPSC amplitude and latency (Right). $\mathrm{P}<0.0001, t_{9}=29.1, \mathrm{n}=10$ and $\mathrm{P}<$ $0.0001, t_{9}=44.2, \mathrm{n}=10$, respectively. D. R26R-FLEX-TVA-2A-RabiesG-2A-tdTomato-FLEX (R26F-RTT) mice before and after Cre recombinase. E-F. Schematic of EnvA G-deleted RabieseGFP virus injection into the brain of $C r e-R 26 F-R T T$ mice, and into the lateral ventricle (SVZ) of Chat-Cre::R26F-RTT mice. G. Immunofluorescence staining for GFP (green) and ChAT (red) in the SVZ and striatum on the injected side. H. Schematic representation of monosynaptic connections of subep-ChAT ${ }^{+}$neurons. All error bars indicate SEM. 


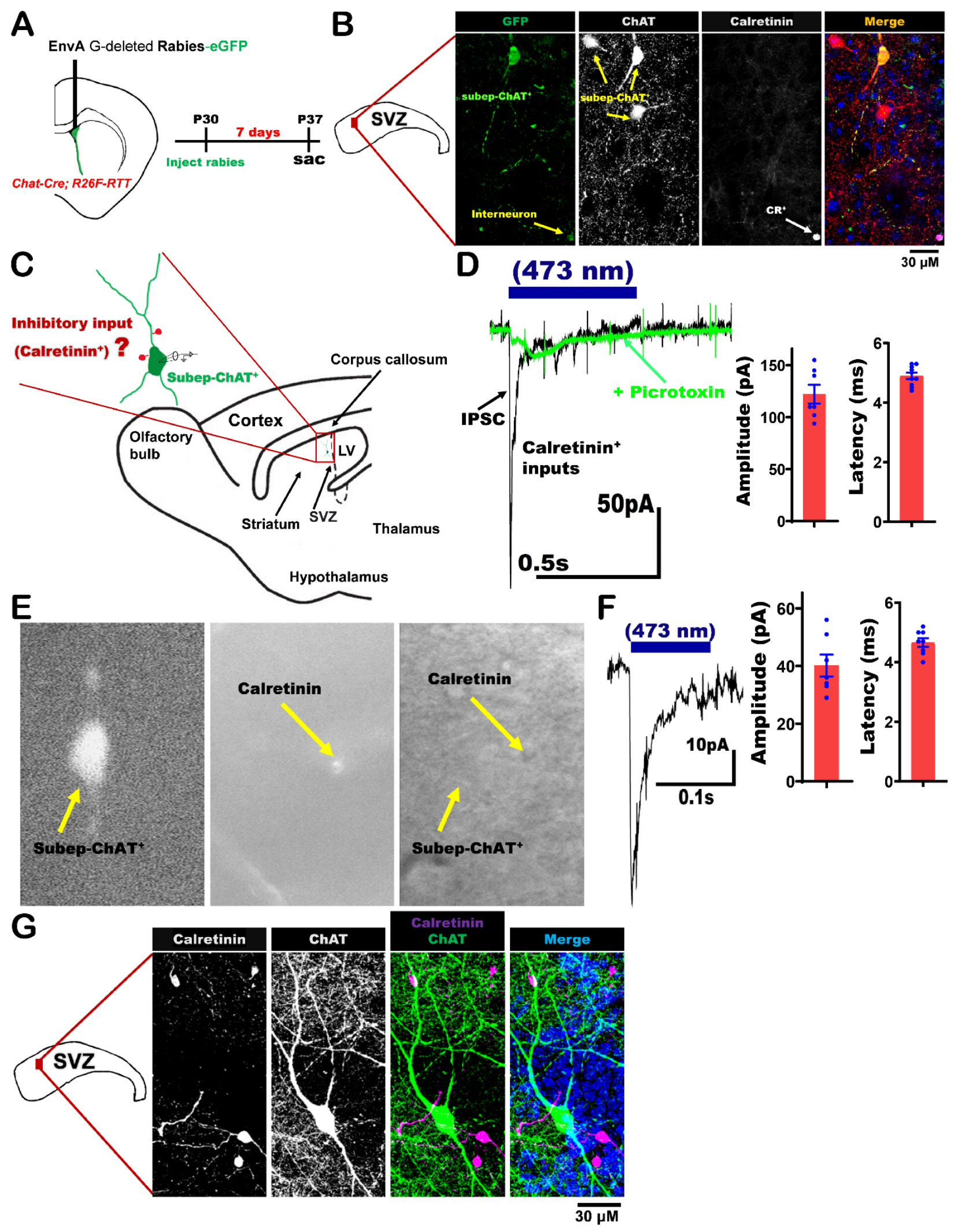


Figure 2. Local calretinin-positive $\left(\mathrm{CR}^{+}\right) \mathrm{GABAergic}$ interneurons provide inhibitory inputs to subep-ChAT ${ }^{+}$neurons. A. Experimental design for injecting the EnvA G-deleted Rabies-eGFP virus to the LV of Chat-Cre::R26F-RTT mice. B. Immunofluorescence staining for GFP (green) and ChAT (red) in the wholemount of the injected side from P37 Chat-Cre::R26F-RTT mice. C. Schema illustrating electrophysiological recording of calretinin-positive $\left(\mathrm{CR}^{+}\right)$inhibitory inputs to the subep-ChAT ${ }^{+}$neurons from P30 Cr-Cre::ChAT-eGFP.:ChR2 mice. D. Evoked IPSCs from subep-ChAT ${ }^{+}$neurons upon photostimulation for $500 \mathrm{~ms}$; it is blocked by the $\mathrm{GABA}_{\mathrm{A}} \mathrm{R}$ antagonist picrotoxin (Left). Average current amplitude and latency of the IPSCs upon stimulation (Right). $\mathrm{P}<0.0001, t_{9}=19.1, \mathrm{n}=10$ and $\mathrm{P}<0.0001, t_{9}=46.9, \mathrm{n}=10$, respectively. E. Representative subep-ChAT ${ }^{+}$neuron recording from P30 Cr-Cre::ChAT-eGFP.:ChR2 wholemount. Left; subep$\mathrm{ChAT}^{+}$neuron (473nm light). Middle; Subep-CR ${ }^{+}$neurons (590nm light). Right; bright field image showing both subep-ChAT ${ }^{+}$and $-\mathrm{CR}^{+}$neurons. F. Evoked IPSCs in subep-ChAT ${ }^{+}$neuron following photostimulation for 100ms (Left). Average current amplitude and latency of the IPSCs upon stimulation (Right). G. Immunofluorescence staining for CR (purple) and ChAT (green) in the wholemount SVZ from $C 57 B L / 6 J$ mice. 

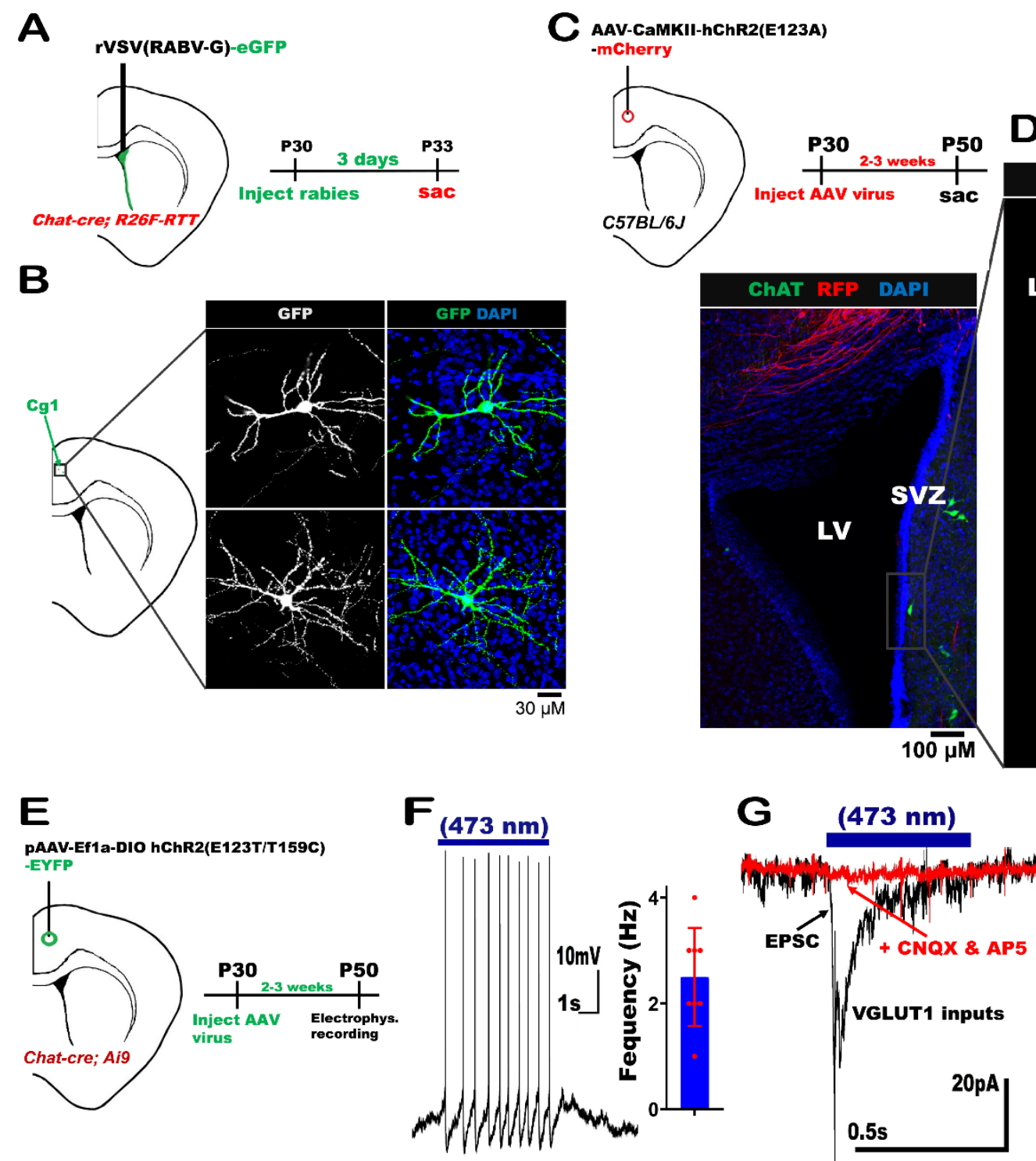

AAV-CaMKII-hChR2(E123A)

-mCherry
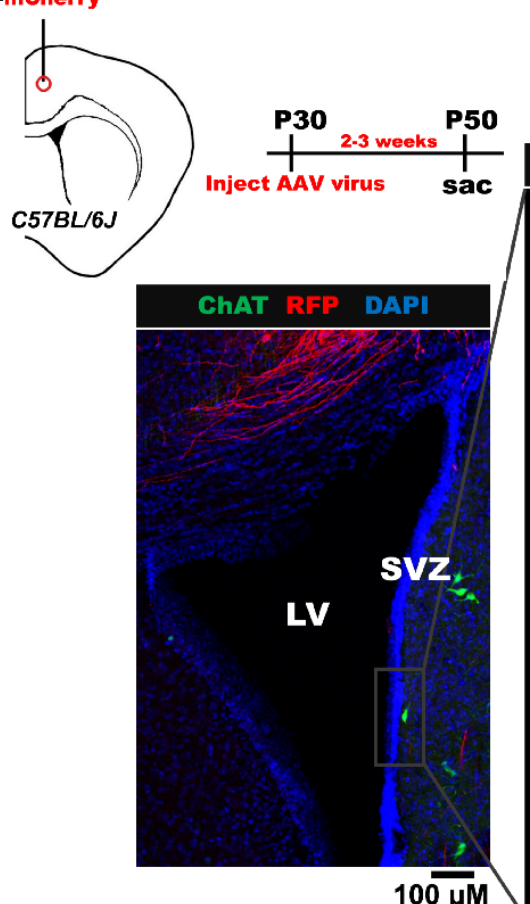

$\mathbf{V}$

SVz

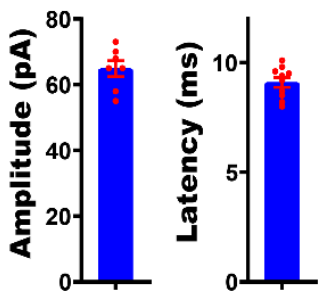

Figure 3. Anterior cingulate cortex projects directly to subep-ChAT ${ }^{+}$neurons. A. Schema illustrating injecting the rVSV(RABV-G)-eGFP virus into the SVZ of P30 Chat-Cre::R26F-RTT mice. B. Immunofluorescence staining for GFP (green) in the ipsilateral $\mathrm{Cg} 1$ region of the anterior cingulate cortex (injection side) from P33 Chat-Cre::R26F-RTT mice. C. Schema showing injection of AAV-CaMKII-hChR2(E123A)-mCherry virus into the $\mathrm{Cg} 1$ of $\mathrm{P} 30 \mathrm{C} 57 \mathrm{BL} / 6 \mathrm{~J}$ mice. D. Immunofluorescence staining for ChAT (green) and infected projections of the Cg1 neurons (red) in the ipsilateral SVZ (injection side) from P50 C57BL/6J mice in panel C. E. Experimental design for injecting pAAV-Ef1a-DIO hChR2(E123T/T159C)-EYFP virus into Cg1 region of Chat-Cre::Ai9 mice. F. Whole cell current clamp recoding of evoked action potentials (APs) from subep-ChAT ${ }^{+}$neurons upon photo-stimulation for $5 \mathrm{~s}$ (Left). Average spike frequency (Right). $\mathrm{P}<$ $0.0001, t_{7}=7.6, \mathrm{n}=8$. G. Electrophysiological recordings of EPSCs were obtained in whole-cell recordings from subep-ChAT ${ }^{+}$neurons upon $473 \mathrm{~nm}$ light stimulation for $500 \mathrm{~ms}$, and after blocking with AMPA and NMDA receptors antagonists (CNQX and AP-5, respectively) (Left). Average EPSC amplitude and latency (Right). All error bars indicate SEM. 


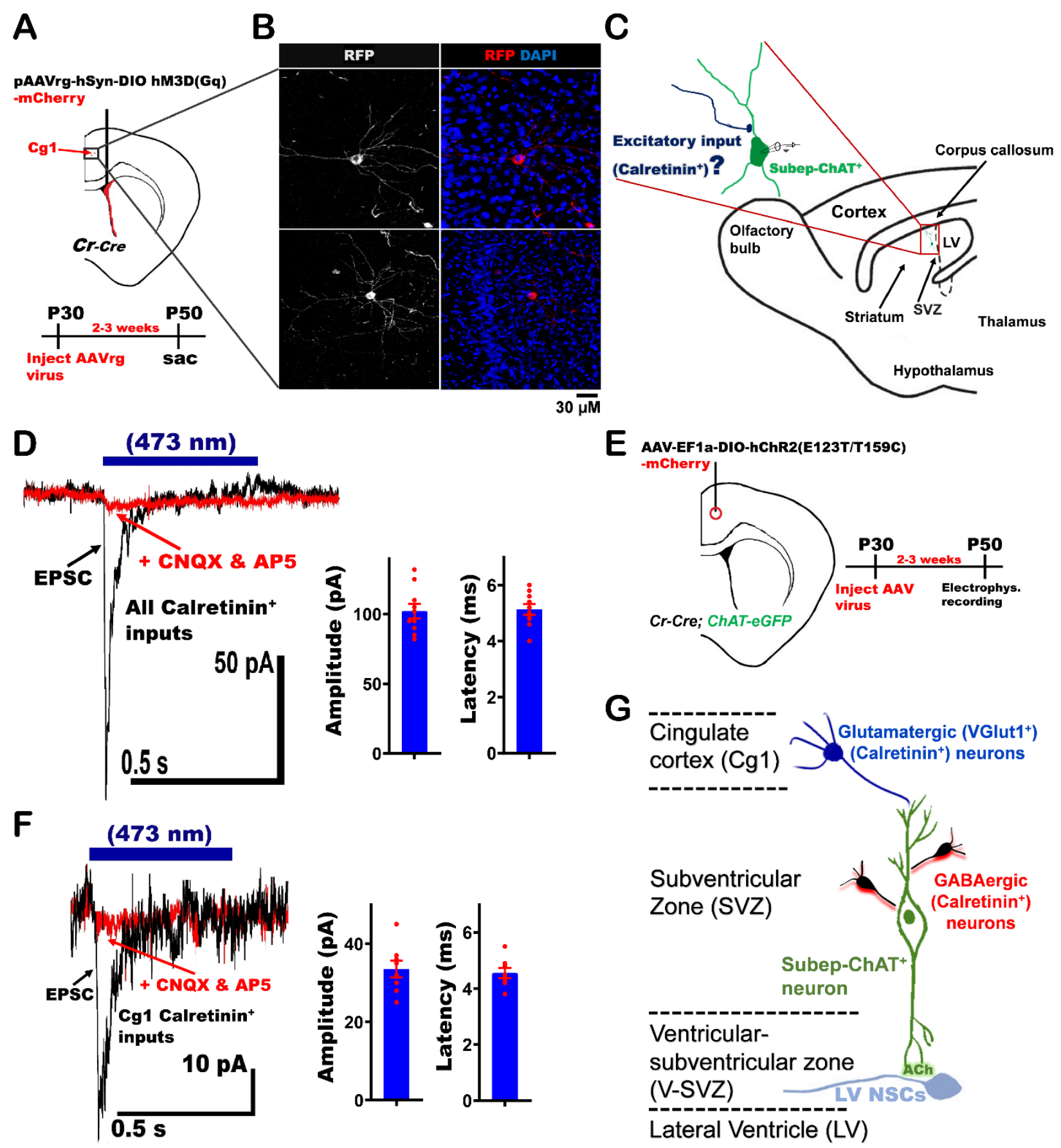

Figure 4. Presynaptic cingulate neurons that excite subep-ChAT ${ }^{+}$neurons are calretininpositive $\left(\mathbf{C R}^{+}\right)$. A. Schematic illustration of the experimental design. pAAVrg-hSyn-DIO $\mathrm{hM} 3 \mathrm{D}(\mathrm{Gq})$-mCherry virus was injected into the SVZ of P30 Cr-Cre mice. B. Immunofluorescence staining for RFP (red) in the ipsilateral Cg1 (injection side) from P50 Cr-Cre mice. C. Schema showing presynaptic excitatory $\left(\mathrm{CR}^{+}\right)$inputs to the subep-ChAT ${ }^{+}$neurons. D. In P30 $\mathrm{Cr}$ Cre::ChAT-eGFP::ChR2 mice, EPSCs were recorded from subep-ChAT ${ }^{+}$neurons upon 473nm light stimulation for 500ms. EPSCs were blocked by AMPA and NMDA antagonists (CNQX and AP-5, respectively) (Left). Average current amplitude and latency (Right). E. Experimental design for injecting AAV-EF1a-DIO-hChR2(E123T/T159C)-EYFP virus into the Cg1 of P30 Cr- 
Cre::ChAT-eGFP mice. F. EPSCs evoked specifically by $\mathrm{Cg} 1 \mathrm{CR}^{+}$input. EPSCs were recorded from subep-ChAT ${ }^{+}$neurons of injected mice in panel E upon 473nm light stimulation for 500ms. EPSCs were blocked by CNQX and AP-5 (Left). An average current amplitude and latency (Right). $\mathrm{P}<0.0001, t_{7}=15.3, \mathrm{n}=8$ and $\mathrm{P}<0.0001, t_{7}=24.9, \mathrm{n}=8$, respectively. G. Schematic summary of circuit regulation of LV qNSCs. 

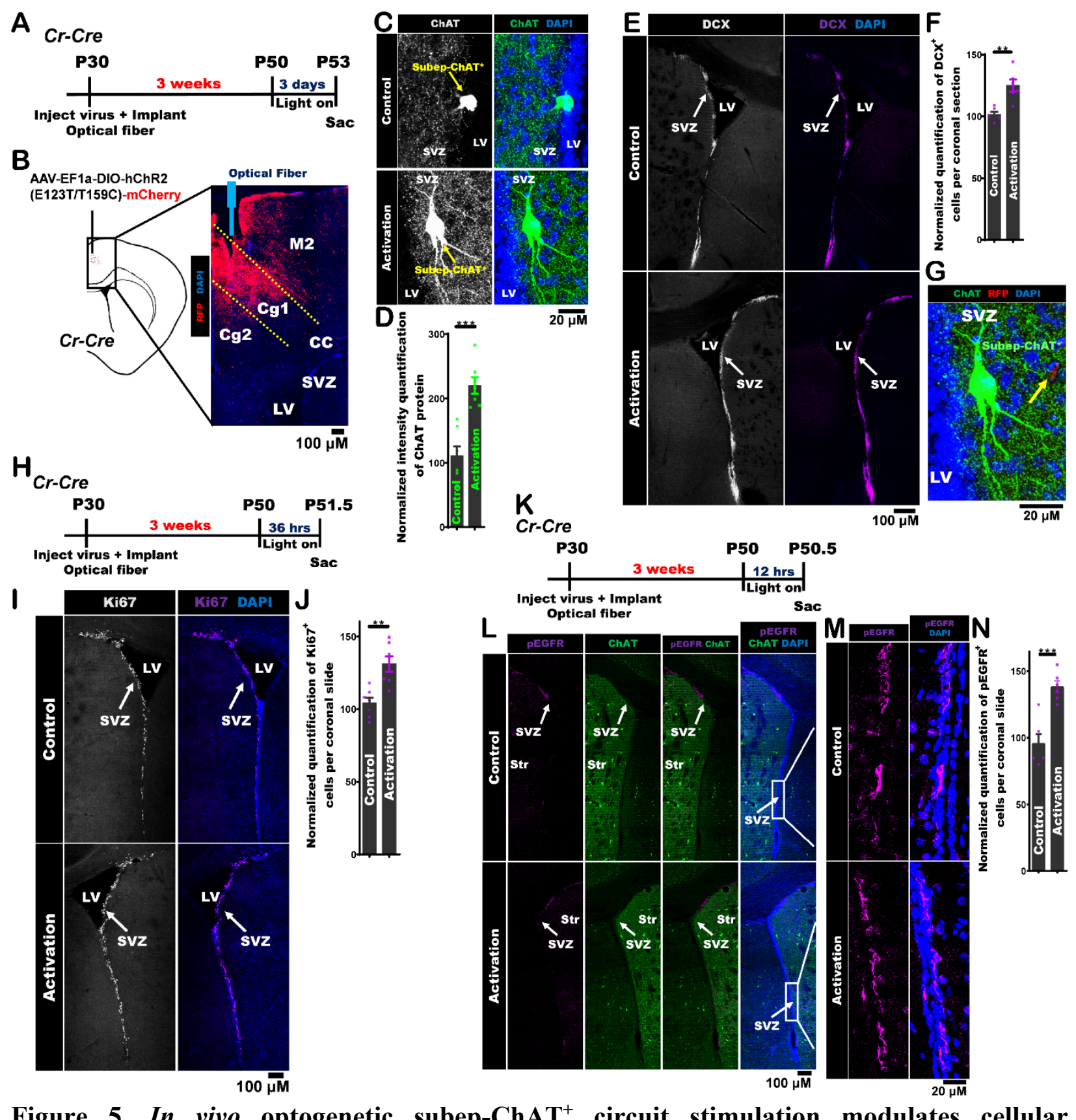

Figure 5. In vivo optogenetic subep-ChAT $\mathrm{T}^{+}$circuit stimulation modulates cellular proliferation and neurogenesis in the SVZ niche. A. The experimental design for in vivo optogenetic stimulation of P53 Cr-Cre mice post injecting AAV-EF1a-DIOhChR2(E123T/T159C)-mCherry virus and implanting optical fiber at P30 into ipsilateral Cg1. B. Immunofluorescence staining for RFP (red) in the ipsilateral (injection side) from P60 $\mathrm{Cr}$-Cre mice. C. ChAT immunofluorescence staining (grey; left images and green; right images) of contralateral (upper images; control) vs. ipsilateral (lower images; activation) sides of SVZ from stimulated coronal section of mice in panel A. Identical settings from same brain section were used for imaging. D. Analysis of ChAT intensity in SVZ of ipsilateral (activation) vs. contralateral (control). $\mathrm{P}<0.0001, \mathrm{n}=7$, Unpaired t-test. E. DCX immunofluorescence staining (grey; left images and purple; right images) of contralateral (upper images; control) vs. ipsilateral (lower images; 
activation) sides of SVZ from stimulated coronal section of mice in panel A. Identical settings from same brain section were used for imaging. F. Analysis of DCX intensity in ipsilateral SVZ (activation) vs. contralateral SVZ (control). $\mathrm{P}=0.0015, \mathrm{n}=6$, Unpaired t-test. G. Immunofluorescence staining for ChAT (green) and infected projections of the Cg1 neurons (red) in the ipsilateral SVZ (injection side) from P60 mice in panel A. H. Experimental design for in vivo optogenetic stimulation of P51.5 Cr-Cre mice. AAV-EF1a-DIO-hChR2(E123T/T159C)mCherry virus and optic fiber was implanted into ipsilateral $\mathrm{Cg} 1$ at P30. I. Ki67 immunofluorescence staining (grey; left images and purple; right images) of contralateral SVZ (upper images; control) vs. ipsilateral SVZ (lower images; activation) from stimulated coronal section of mice in panel $\mathrm{H}$. Identical settings from same brain section were used for imaging. $\mathbf{J}$. Analysis of Ki67 intensity in SVZ of ipsilateral (activation) vs. contralateral (control). P = 0.0013, $\mathrm{n}=7$, Unpaired t-test. K. The experimental design for in vivo optogenetic stimulation of P50.5 $\mathrm{Cr}$ Cre mice post injecting AAV-EF1a-DIO-hChR2(E123T/T159C)-mCherry virus and implanting optical fiber at $\mathrm{P} 30$ into ipsilateral $\mathrm{Cg} 1$. L-M. Immunofluorescence staining for pEGFR(PhosphoY1068) (purple) and ChAT (green) of contralateral SVZ (upper images; control) vs. ipsilateral SVZ (lower images; activation) sides from stimulated coronal section of mice in panel A. Identical settings from same brain section were used for imaging. Identical settings from same brain section were used for imaging. N. Analysis of $\mathrm{pEGFR}^{+}$cells in SVZ of ipsilateral (activation) vs. contralateral (control). ${ }^{* * *} \mathrm{P}$ value $=0.0003, \mathrm{n}=6$, Unpaired t-test; bars=S.E.M. 


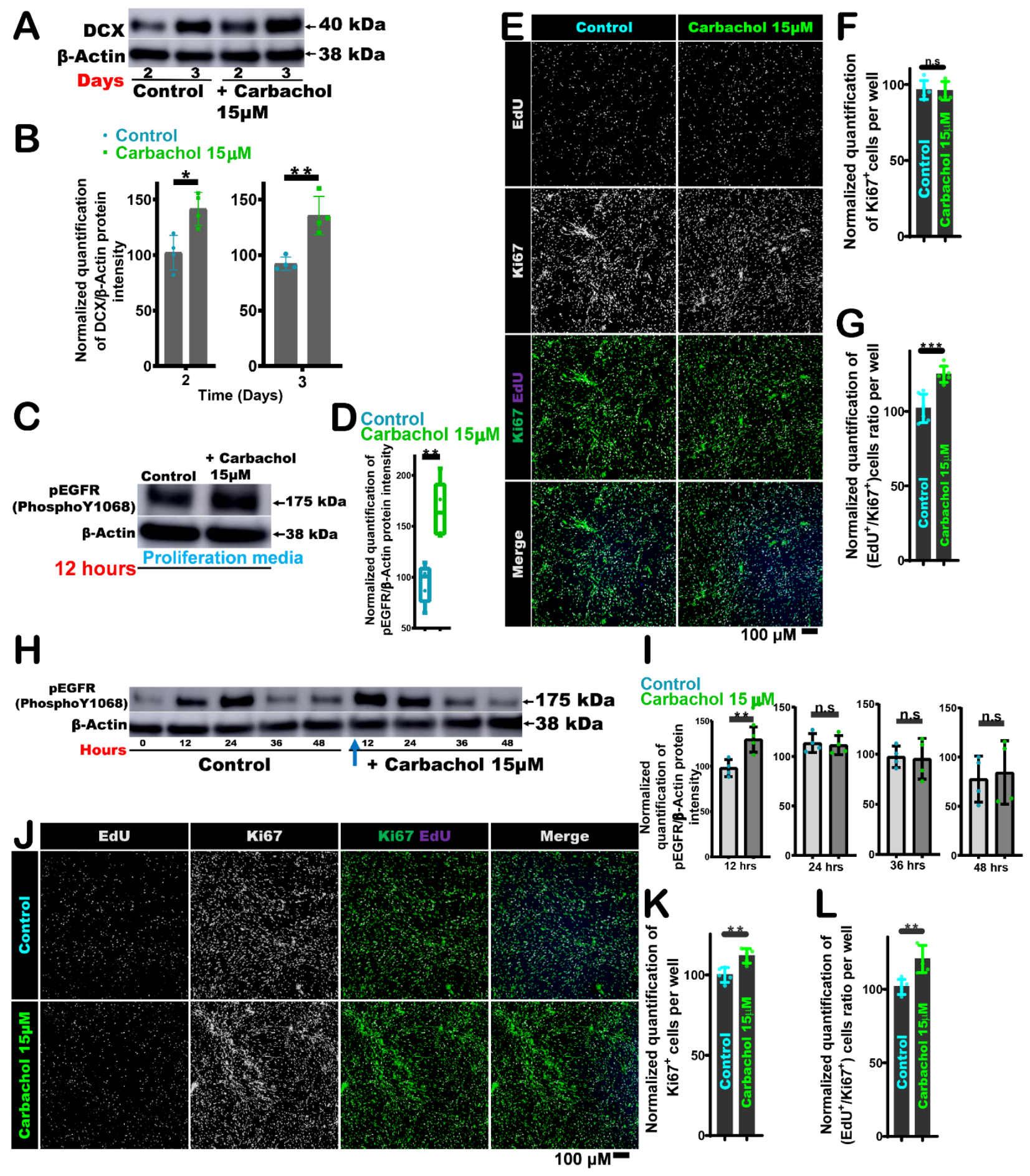

Figure 6. ACh modulates the initial phase of NSCs proliferation and neurogenesis in SVZ NSCs culture. A. Western blot detection of DCX in Control and treatment samples with carbachol $(15 \mu \mathrm{M})$ of SVZ NSCs cultures in differentiation media collected post the second and third days. B. Analysis normalized quantification of $\mathrm{DCX} / \beta$-actin protein intensity for control and treated samples with carbachol from western plot in panel A. $* \mathrm{P}=0.0107$ and $* * \mathrm{P}=0.0031$ for days two and three, respectively, $\mathrm{n}=4$, Unpaired t-test. C. Western blot detection of pEGFR 
(PhosphoY1068) in control and treated samples with carbachol $(15 \mu \mathrm{M})$ of SVZ NSCs cultures in proliferation media collected after 12 hours. D. Analysis of normalized quantification of pEGFR(PhosphoY1068)/ $\beta$-actin protein intensity in control and treated samples with carbachol $(15 \mu \mathrm{M})$ of SVZ NSCs cultures from panel C. $* * \mathrm{P}=0.0012, \mathrm{n}=5$, Unpaired t-test. E. Immunofluorescence staining for EdU (purple) and Ki67 (green) in control and treated samples with carbachol $(15 \mu \mathrm{M})$ of SVZ NSCs cultures in proliferation media collected after one day. F. Analysis of normalized quantification of $\mathrm{Ki}^{+} 7^{+}$cells per well in control and treated samples with carbachol $(15 \mu \mathrm{M})$ of SVZ NSCs cultures from panel E. $\mathrm{P}=$ n.s., $\mathrm{n}=5$, Unpaired t-test. G. Analysis of normalized quantification of $\left(\mathrm{EdU}^{+} / \mathrm{Ki}^{+} 7^{+}\right) / \beta$-actin cells ratio per well in control and treated samples with carbachol $(15 \mu \mathrm{M})$ of SVZ NSCs cultures from panel $\mathrm{E}$. $* * * \mathrm{P}=0.0005, \mathrm{n}=6$, Unpaired t-test. H. Western blot detection of pEGFR(PhosphoY1068)/ $\beta$-actin in control and treated samples with carbachol $(15 \mu \mathrm{M})$ of SVZ NSCs cultures in differentiation media collected every 12 hours for two days. I. Analysis of normalized quantification of pEGFR(PhosphoY1068)/ $\beta$-actin protein intensity in control and treated samples with carbachol $(15 \mu \mathrm{M})$ of SVZ NSCs cultures from panel H. For sample collected after 12, 24, 36 and $48 \mathrm{hrs}$. **P $=0.0095(12 \mathrm{hrs})$ and $\mathrm{P}=$ n.s. $(24,36$ and $48 \mathrm{hrs}), \mathrm{n}=4$, Unpaired t-test. J. Immunofluorescence staining for EdU (purple) and Ki67 (green) in control and treated samples with carbachol $(15 \mu \mathrm{M})$ of SVZ NSCs cultures in differentiation media collected after one day. K. Analysis of normalized quantification of $\mathrm{Ki}^{+} 7^{+}$cell per well in control and treated samples with carbachol $(15 \mu \mathrm{M})$ of SVZ NSCs cultures in differentiation media collected after 12 hours. $* * \mathrm{P}=0.0032, \mathrm{n}=5$, Unpaired $\mathrm{t}$ test. L. Analysis of normalized quantification of $\left(\mathrm{EdU}^{+} / \mathrm{Ki} 67^{+}\right) / \beta$-actin cells ratio per well in control and treated samples with carbachol $(15 \mu \mathrm{M})$ of SVZ NSCs cultures from panel $\mathrm{K}$. **P = $0.0040, \mathrm{n}=5$, Unpaired t-test. 

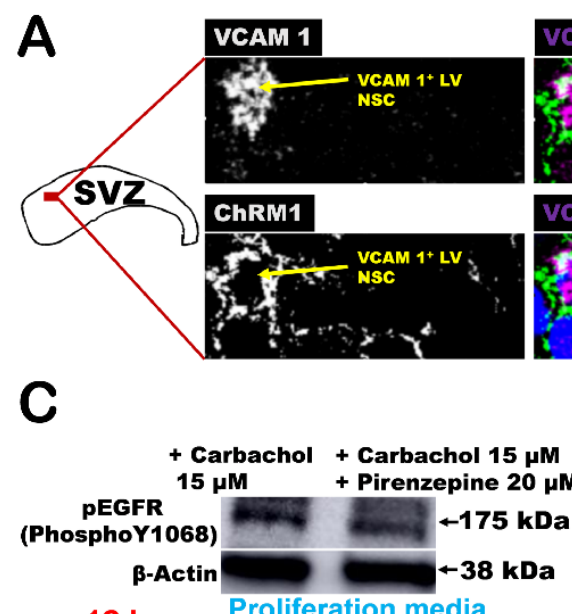

12 hours Proliferation media
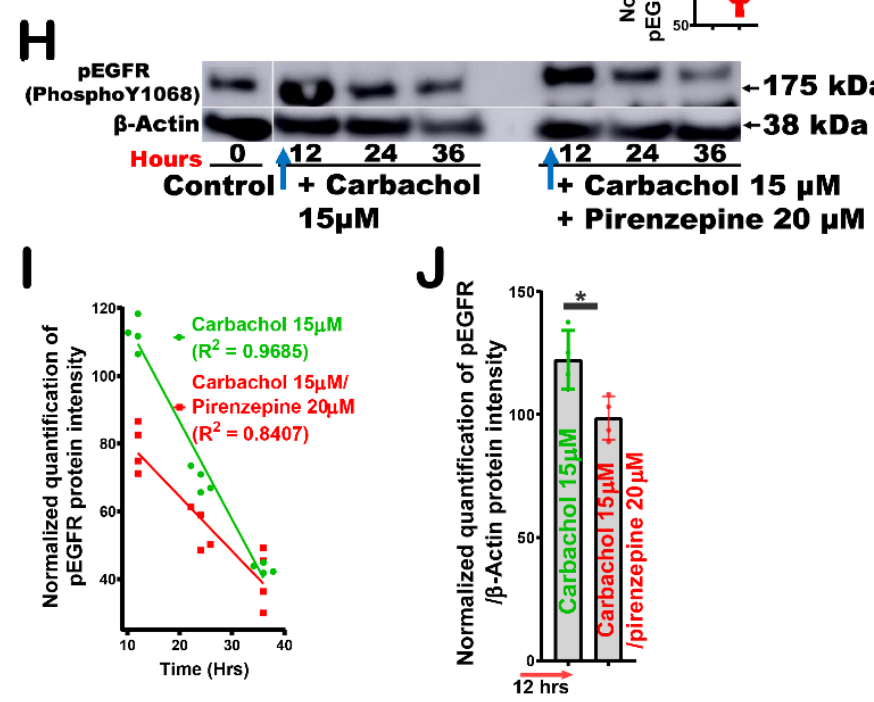

Pirenzepine 2q,M

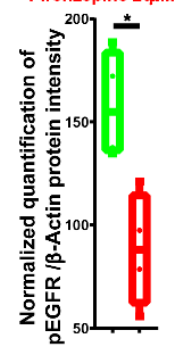

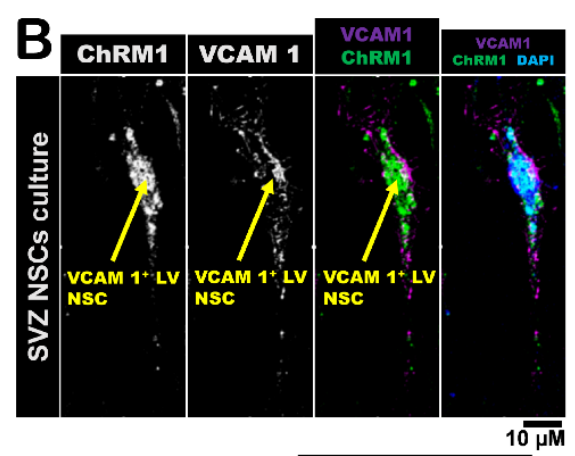

-175 kDa
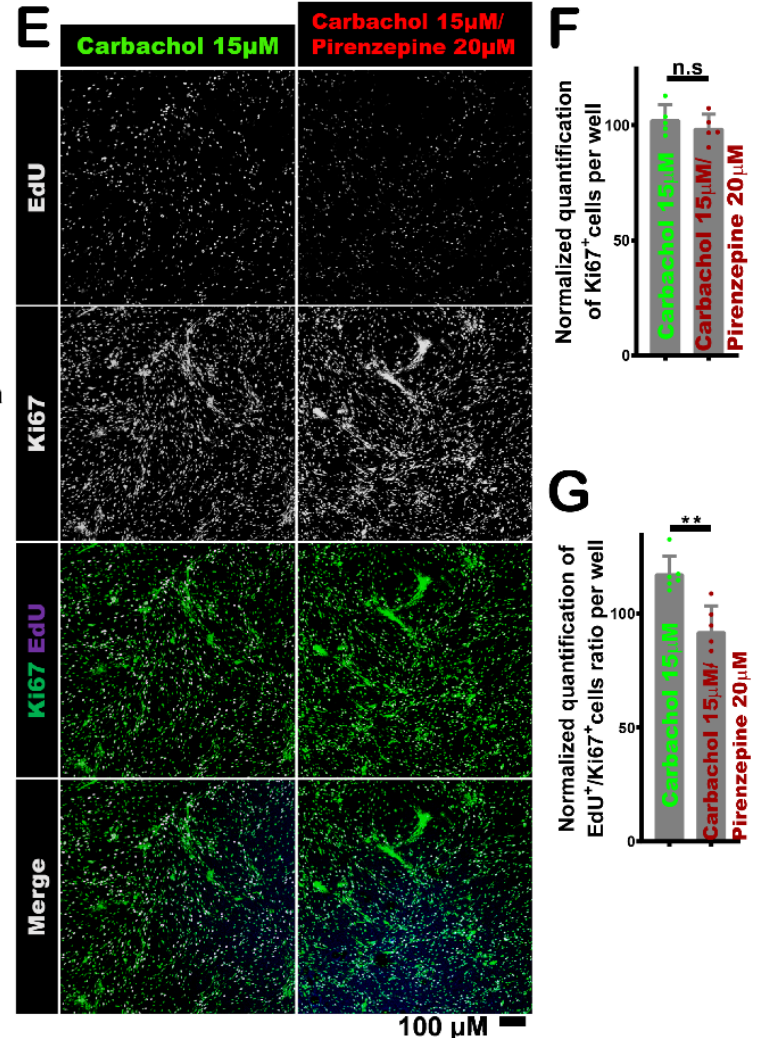

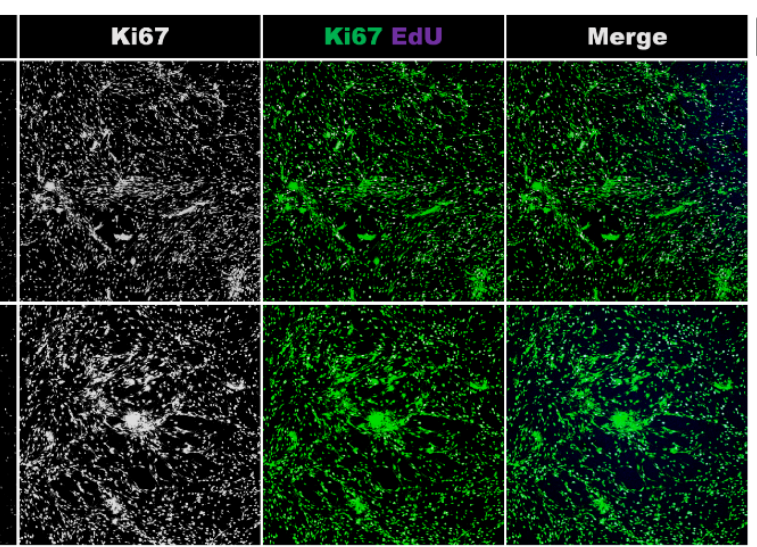

$100 \mu \mathrm{M}=$
M

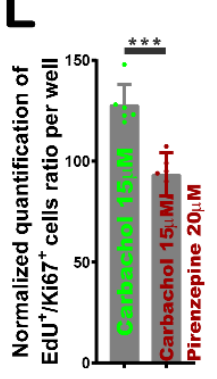

Subventricular Zone (SVZ)

Subep-ChAT neuron

Ventricular-

subventricular zone (V-SVZ)

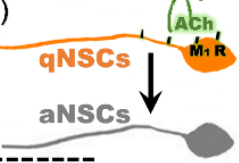

Lateral Ventricle (LV) 
Figure 7. Muscarinic 1 receptor (M1) activation regulates the activity of qNSCs in SVZ niche. A. Immunofluorescence staining for VCAM 1 (Purple) and ChRM1 (green) in the SVZ niche from P30 C57BL/6J mice. B. Immunofluorescence staining for VCAM 1 (Purple) and ChRM1 (green) from the SVZ NSCs culture. C. Western blot detection of pEGFR (PhosphoY1068) in treated samples with carbachol $(15 \mu \mathrm{M})$ and carbachol $(15 \mu \mathrm{M})$ /pirenzepine $(20 \mu \mathrm{M})$ of SVZ NSCs cultures in proliferation media collected after 12 hours. D. Analysis of normalized quantification of pEGFR(PhosphoY1068)/ $\beta$-actin protein intensity in treated samples with carbachol $(15 \mu \mathrm{M})$ and carbachol $(15 \mu \mathrm{M}) /$ pirenzepine $(20 \mu \mathrm{M})$ of SVZ NSCs cultures from panel C. $* \mathrm{P}=0.0107, \mathrm{n}=4$, Unpaired t-test. E. Immunofluorescence staining for EdU (purple) and Ki67 (green) in treated samples with carbachol $(15 \mu \mathrm{M})$ and carbachol $(15 \mu \mathrm{M})$ /pirenzepine $(20 \mu \mathrm{M})$ of SVZ NSCs cultures in proliferation media collected after one day. F. Analysis of normalized quantification of $\mathrm{Ki}^{+} 7^{+}$ cells per well in treated samples with carbachol $(15 \mu \mathrm{M})$ and carbachol $(15 \mu \mathrm{M}) /$ pirenzepine $(20$ $\mu \mathrm{M}$ ) of SVZ NSCs cultures from panel E. $\mathrm{P}=\mathrm{n} . \mathrm{s} ., \mathrm{n}=5$, Unpaired t-test. G. Analysis of normalized quantification of $\mathrm{EdU}^{+} / \mathrm{Ki} 67^{+}$cells ratio per well in control and treated samples with carbachol $(15 \mu \mathrm{M})$ of SVZ NSCs cultures from panel E. $* * \mathrm{P}=0.0012, \mathrm{n}=6$, Unpaired t-test. H. Western blot detection of pEGFR(PhosphoY1068)/ $\beta$-actin in treated samples with carbachol $(15 \mu \mathrm{M})$ and carbachol $(15 \mu \mathrm{M}) /$ pirenzepine $(20 \mu \mathrm{M})$ of SVZ NSCs cultures in differentiation media collected every 12 hours for 36 hours. I. Analysis of normalized quantification of pEGFR(PhosphoY1068)/ $\beta$-actin protein intensity in treated samples with carbachol $(15 \mu \mathrm{M})$ and carbachol $(15 \mu \mathrm{M}) /$ pirenzepine $(20 \mu \mathrm{M})$ of SVZ NSCs cultures from panel $\mathrm{H}$. $\mathrm{P}<0.0001$ for both, $\mathrm{n}=4$, linear regression analysis shows a significant relationship between intensity and time. $\mathbf{J}$. Analysis of normalized quantification of pEGFR(PhosphoY1068)/ $\beta$-actin protein intensity in treated samples with carbachol $(15 \mu \mathrm{M})$ and carbachol $(15 \mu \mathrm{M})$ /pirenzepine $(20 \mu \mathrm{M})$ of SVZ NSCs cultures in differentiation media collected after 12 hours. $* \mathrm{P}=0.0183, \mathrm{n}=4$, Unpaired t-test. $\mathbf{K}$. Immunofluorescence staining for EdU (purple) and Ki67 (green) in treated samples with carbachol $(15 \mu \mathrm{M})$ and carbachol $(15 \mu \mathrm{M}) /$ pirenzepine $(20 \mu \mathrm{M})$ of SVZ NSCs cultures in differentiation media collected after one day. L. Analysis of normalized quantification of $\left(\mathrm{EdU}^{+} / \mathrm{Ki}^{+} 7^{+}\right) / \beta$-actin cells ratio per well in treated samples with carbachol $(15 \mu \mathrm{M})$ and carbachol $(15 \mu \mathrm{M}) /$ pirenzepine $(20 \mu \mathrm{M})$ of SVZ NSCs cultures from panel K. $* * * \mathrm{P}=0.0012, \mathrm{n}=6$, Unpaired t-test. M. Schematic summary of subep-ChAT ${ }^{+}$neuron activation of LV qNSCs. All errors bars indicate SEM. 